\title{
Recent Advances in the Laser Radiation Transport through the Head Tissues of Humans and Animals - A Review
}

\author{
Alaa Sabeeh ${ }^{1 *}$ and Valery V. Tuchin ${ }^{1,2,3+}$ \\ ${ }^{1}$ Research-Educational Institute of Optics and Biophotonics, Saratov State University, 83 Astrakhanskaya str., \\ Saratov 410012, Russia \\ ${ }^{2}$ Interdisciplinary Laboratory of Biophotonics, National Research Tomsk State University, 36 Lenin Ave., \\ Tomsk 634050, Russia \\ ${ }^{3}$ Laboratory of Laser Diagnostics of Technical and Living Systems, Institute of Precision Mechanics and Control of the \\ Russian Academy of Sciences, 24 Rabochaya str., Saratov 410028, Russia \\ e-mail: * alaa2015krote@yahoo.com, + tuchinvv@mail.ru
}

\begin{abstract}
Modern studies of the penetration of light into biological tissues is becoming very important in various medical applications. This is an important factor for determining the optical dose in many diagnostic and therapeutic procedures. The absorption and scattering properties of the tissue under study determine how deeply the light will penetrate into the tissue. However, these optical properties are highly dependent on the wavelength of the light source and tissue condition. This overview paper analyzes the transmission of light through different areas of human and animal head tissues, and the optimal laser wavelength and power density required to reach different parts of the brain are determined using lasers with different wavelengths by comparing the distribution of fluence, penetration depth and the mechanism of interaction between laser light and head tissues. The power variation in different regions of the head is presented, as estimated using Monte Carlo (MC) simulations. Data are analyzed for the absorption and scattering coefficients of the head tissue layers (scalp, skull, brain), calculated using integrating sphere measurements and inverse problem solving algorithms such as inverse MC (IMC) and adding-doubling (IAD). This study not only offered a quantitative comparison between wavelengths in terms of light transmission efficiency, but also anticipated the exciting opportunity for online, accurate and visible optimization of LLLT lighting parameters. (C) 2020 Journal of Biomedical Photonics \& Engineering.
\end{abstract}

Keywords: transcranial laser irradiation; tissue scattering; optical transmission; tissue optics; photothermal effects; nonlinear interactions; temperature; tissue damage; photochemical processes; PDT; LLLT.

Paper \#3393 received 15 Nov 2020; revised manuscript received 18 Dec 2020; accepted for publication 18 Dec 2020; published online 31 Dec 2020. doi: 10.18287/JBPE20.06.040201.

\section{Introduction}

In this year, 2020, the laser celebrates its $60^{\text {th }}$ anniversary. While in the beginning, it was a solution looking for a problem; it is meanwhile widely spread in industry, science, medicine. Understanding the fundamentals of laser light, the optical properties of biological tissues and light-tissue interaction, is essential for all health professionals wishing to work with lasers. We will present some important factors that directly or indirectly affect the interaction of light with tissues and we will discuss how some characteristics of laser light and the optical properties of biological tissues and matter, can govern their interaction. The recently accelerated new discoveries in the field of lasers, have stimulated the rising interest observed in the science of biophotonics $[1,2]$. 
Utilization of low-level laser in biological and medical applications of treating diseases non-invasively is gaining validity. Infrared laser, in particular, is widely applied for its potential to interact with deeper tissues. The transmission of laser radiation through the head is position and dose dependent. The maximum laser transmission was recorded at the frontal part of the animals (for example, rat) head, while the minimum transmission was recorded at the back of the head and this at all the laser irradiation doses. This result could be attributed to the difference in skull thickness along the head axis. The back of the skull recorded the highest thickness and therefore the minimum laser transmission. These results were evidenced by the transmission profiles obtained by the skull only. Tissue scattering properties are mainly affected by the structure of the tissue components such as cell membrane and organelles and highly dependent on the wavelength of the incident light. However, recent studies revealed that tissue scattering can also be affected by the intensity of the utilized laser light and its spot size as well [3].

In this paper, we will discuss several recent experiences, including the optical permeability of the human and animal head tissues, such as brain cortex, cranial bone and skin which has been estimated, aiming to the transcranial light applications, such as brain imaging or therapy. The transmission and absorption spectral measurements were carried out in the broad spectral range from 350 to $2800 \mathrm{~nm}$ (see Fig. 1).
Optical attenuation coefficients have been determined for all optical transparency windows of the investigated tissues. The authors of Refs. [4, 5] showed for the first time the distinct existence of SWIR-II for skin, subcutaneous fat and bone tissues, and it can be assumed that this window has a significant potential for the transcranial light applications. Also measurements of the optical properties (diffuse reflectance, total and collimated transmittance) of brain tissues in healthy rats and rats with C6-glioma were performed in the spectral range from 350 to $1800 \mathrm{~nm}$ [6] It was obtained that the 10 day development of glioma led to increase of absorption coefficient, which was associated with the water content elevation in the tumor. However, further development of the tumor (formation of the necrotic core) led to decrease in the water content. Light penetration depth into the healthy and tumor brain was evaluated [6]. Study [7] attempts to quantitatively revisit this issue by innovative high-realistic 3D Monte Carlo (MC) modeling of stimulated light penetration with a high precision for Chinese human head. Authors made straightforward and quantitative comparison among the effects by the light properties for different wavelengths as well as different beams, which are in the range of light treatments. This study offered a quantitative guide for optimization of the light stimulation parameters for medical treatments [7].

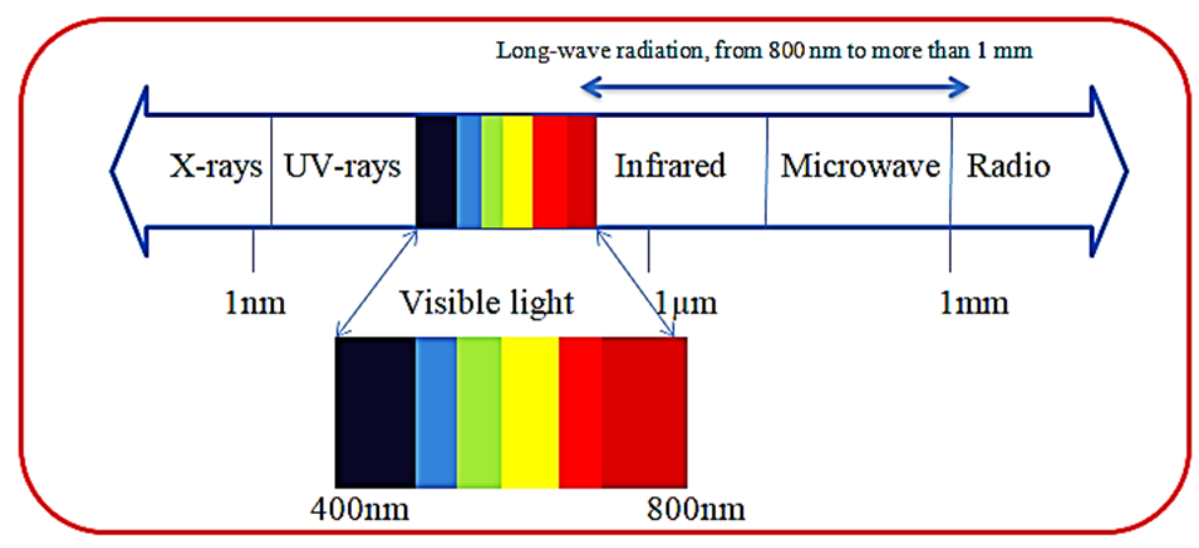

Fig. 1 Electromagnetic radiation of the broad spectral range used in biomedical applications.

\section{Biological tissues}

Biological tissue is an aggregate of similar cells and cell products (tissue fibers and gels) forming a definite kind of structural material in normal or abnormal state [8]. There are three major types of tissues, such as epithelial, connective, and glandular. More specifically tissues characterized as areolar, reticular, fibrous, tubular, and elastic. There is wide variety of structures within cells that determine tissue light scattering. Cell nuclei are on the order of $5-10 \mu \mathrm{m}$ in diameter; mitochondria, lysosomes and peroxisomes have dimensions of $1-2 \mu \mathrm{m}$; ribosomes are on the order of $20 \mathrm{~nm}$ in diameter; and structures within various organelles, such as mitochondria, can have dimensions up to a few hundred nanometers. It can be divided into soft tissues and hard tissue.

Where a soft tissue has its structural peculiarities and elasticity providing the specificity of light-tissue interaction. Tissue morphology, especially sizing and shaping of tissue constitutions (particles), and particle material (molecular structure) are important. For some tissues, the size distribution of the particles may be essentially monodisperse and for others it may be very broad.

The brain and spinal cord together make up the central nervous system. Grey matter is a nervous tissue found in the central nervous system. Brain nuclei and nerve centers are composed of grey matter and 
coordination in the central nervous system is affected in grey matter. White matter is also a nervous tissue found in the central nervous system.

The brain and spinal cord are protected by three layers of tissue, called spinal meninges that surround them. The dura mater is the outermost layer, and it forms a tough protective coating. Between the dura mater and the surrounding bone of the skull or vertebrae is a space, called the epidural space, which is filled with adipose tissue, and it contains a network of blood vessels. The arachnoid is the middle protective layer, the space between the arachnoid and the underlying pia mater contains cerebrospinal fluid (CSF). The pia mater is the innermost protective layer, which is very delicate and tightly associated with the surface of the brain and spinal cord. Dura mater is the tough and inflexible two layered tissue. A superficial layer is actually the bone inner periosteum, and a deep layer is the dura mater proper. Dura mater proper consists of layers of densely packed collagen fibrils having an alternating orientation.

While hard tissues differ from it in that bone cells are interconnected by thin protoplasmic processes located in narrow channels in the bone, and feed on the bloodstream. This vascular nature of bone distinguishes it from cartilage. The flexible tissue found in the hollow interior of bones is the bone marrow. From through the analysis of tissue properties, turns out many biological tissues are structurally anisotropic. Tissue birefringence results primarily from the linear anisotropy of fibrous structures, which forms the extracellular media [8]. The refractive index of a medium is higher (speed of light is lower) along the length of fibers than across them. The CSF is the clear liquid that fills the cavities of the brain and spinal cord and the spaces between the arachnoid and pia matter. The fluid moves in a slow current down the central canal and up the spinal meninges.

\section{Neural action}

\subsection{Basic structure of a nerve cell}

Nerve cells, the main constituents of the central nervous system, are elongated cells consisting of the cell body, dendrites, and axon [9, 10]. A complex network of neurons throughout the body allows for the propagation of information that is facilitated by the firing or not firing of neurons, the measure of a neuron's action potential. The biophysical mechanism of action potential in neurons is conventionally understood. In order for a neuron to fire, the resting potential must be raised to the requisite threshold potential. This is mediated by the gradient of electrically charged ions distributed across the cell membrane (see Fig. 2).

There has recently been some suggestion that cellular communication and even possibly neural signaling might make use of biophotons in addition to more wellestablished mechanisms such as neurotransmitters. As noted in a recent paper on the subject, it is known that biophotons are produced in brains [9]. Correlations have been found between biophoton intensity and neural activity as well as oxidative dysfunction of neural cells in rat and mouse brains. There has even been some attempt to explain human intelligence in the context of biophoton emission. Following on from a study that demonstrated that glutamate, a key neurotransmitter, can mediate biophoton production. There is a correlation between higher order intelligence and the spectral redshift of biophotons emitted by sample brain slices. Neurons are said to be well suited for photonics, and there is an evidence that nerve cells contain possible sources of photons from mitochondrial respiration or lipid peroxidation, as well as photon detectors such as centrosomes and chromophores. This suggests that the myelin-coated axons of nerve cells act as waveguides for biophotons and that this could contribute to quantum effects such as entanglement [10].

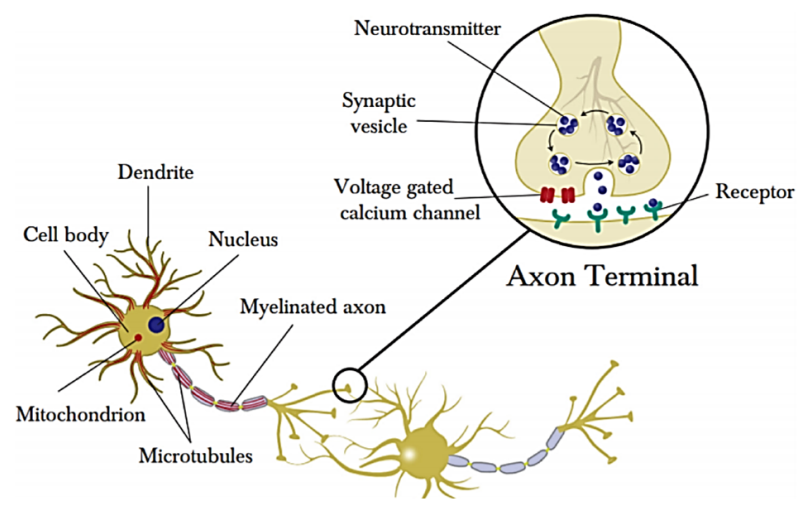

Fig. 2 Basic structure of a nerve cell. Although a number of organelles are missing, this simplified schematic is sufficient to gain some understanding of the location of the various quantum effects discussed in this review. Of interest are the microtubules, which occur in different arrangements throughout the nerve cell and the mitochondria that will be discussed in the context of electron transfer. The myelin insulated axon has also been proposed as a wave guide for neural photonics. The meeting of dendritic spine and axon terminal at the synaptic cleft is enlarged for clearer understanding of the mechanism of neurotransmission and the transfer of neural signals. Synaptic vesicles are also the site of the proposed uptake of Posner molecules during endocytosis Reproduced from Ref. [10] with the permission of AIP Publishing.

Nervous tissues comprise the neurons and neuroglia forming the central nervous system and the peripheral nervous system. The structure of the nervous tissue is soft and possesses a certain level of elasticity that specifies the interaction with the optical waves. The six-layeredcerebral cortex is populated by cholinergic neurons where neurotransmitter acetylcholine $(\mathrm{ACh})$ is mainly utilized for the chemical communications. New theory has been proposed on the manner of biophoton propagation along the axon, and is inspired from the properties of optical communications. In the light-based optical communication, there are three major elements required, namely, source, detector, and directed light propagation medium/waveguide. Inside the living 
organism, in this case human brain, a biophoton is dubbed as the natural light excited during the internal biological process of mitochondrial respiration or lipid oxidation. Meanwhile, mitochondrial centrosomes or chromophores serve as the biophoton detectors [11].

\subsection{Deep-learning-based whole-brain imaging}

Decades of studies have shown that neural connectivity holds the key to brain function. Deciphering neural circuits across the entire brain is central to better understanding of the brain mechanism. With the advancement of fluorescent labeling techniques, can focus on specific neural circuits. The axon from the cell body is about $1 \mu \mathrm{m}$ in diameter, but its length can extend to a few millimeters [12]. Optical clearing methods allow light to penetrate deep into the tissue with less absorption and scattering, thereby extending the imaging depth.

The data acquisition of Golgi-stained mouse brain at optical resolution has been demonstrated using microoptical sectioning tomography (MOST) technologies employing imaging and sectioning simultaneously [12]. A fluorescence MOST (fMOST) system that substituted confocal fluorescence imaging for reflection imaging showed long-range projections of single axons in the Thy1 transgenic (Thy1-eYFP-H and Thy1-GFP-M) mouse brains.

DL-fMOST is a novel deep-learning-based wholebrain imaging method, high throughput and highresolution [12]. Utilized a wide-field microscope for imaging, convolutional neural network $(\mathrm{CNN})$ for realtime optical sectioning, and histological sectioning for exceeding the imaging depth limit and to achieve wholebrain mapping. A 3D dataset of a mouse brain with a voxel size of $0.32 \times 0.32 \times 2 \mu \mathrm{m}$ was acquired in 1.5 days.

Demonstrated the robustness of DL-fMOST for mouse brains with the labeling of different types of neurons. The DL-fMOST method generates a highquality whole-brain dataset at a submicron voxel size and enables counting of cell distribution and tracing neuronal morphology. Another benefit brought by deep learning is the capacity to suppress noise and remove artifacts, which means can directly use the raw data without cumbersome post-processing. To test the applicability of DL-fMOST to different types of neuronal morphology and distributions, reconstructed four whole-brain datasets with various fluorescence-labeled neuron types using the SIM algorithm and CNN prediction. Directly used the Thyl-GFP-M trained model for inference without retraining or transfer learning. There are significant characteristic differences in the neuron morphology and distribution patterns among the four samples.

\section{Light interaction with tissues}

\subsection{Multiple scattering}

Light tissue interaction on the level of a single scattering occurs when a wave undertakes no more than one collision with particles of the medium in which it propagates [13]. For example, a tissue slice which is sufficiently thin single scattering approximation accurately estimates the reflection and transmission of the slab. In contrast, in many real situations, especially in in vivo studies of skin, breast, brain and etc., a multiple scattering - a scattering process in which on average each photon undertakes many scattering events, is more likely.

\subsection{Refractive index and controlling of light interaction with tissues}

Index of refraction of tissue compounds is of great importance for light tissue interaction [13]. This phenomenon is very useful for improvement of facilities of optical tomography and for getting more precise spectroscopic information from the depth of a tissue. Optical parameters of a tissue, in particular refractive index, in the visible and NIR are known to depend on water content. The optical immersion technique is based on the impregnation of a tissue by a biocompatible chemical agent, which may have hyperosmotic properties. The refractive index matching is manifested in the reduction of the scattering coefficient $\left(\mu_{s} \rightarrow 0\right)$ and increase of single scattering directness $(g \rightarrow 1)$. Tissue swelling may take place that could be considered as a competitive process in providing tissue optical clearing. The optical clearing process in collagen-based tissues may involve a change in the supramolecular structure.

\subsection{Photothermal, photoacoustic and acoustic interactions}

When photons traveling in a tissue are absorbed, heat is generated. In its turn, heat induces several effects in tissue that can be presented in the order of amount of heat deposition: temperature increase and reversible and irreversible alterations in tissue structure [14].

The time dependent heat generation in a tissue at interaction with optical radiation is known as optothermal (OT) effect. This interaction also induces a number of thermoelastic effects in a tissue in particular causes generation of acoustic waves. Detection of acoustic waves is a basis for optoacoustic or (photoacoustic) method. The informative features of this method allow one to estimate tissue thermal, optical and acoustical properties which depend on tissue structure [14].

Acoustic wave and light interaction within a tissue as a heterogeneous medium is the basis for acoustooptical tomography (AOT) or ultrasound modulated optical tomography, where the acoustic (ultrasound (US)) modulation of coherent laser light traveling in tissue is provided [13].

US studies are important for AOT development and for quantitative estimation of head US transmittance. In Ref. [15], results of measured acoustic transmission factors through 13 excised skulls of male SpragueDawley rats weighing between 90 and $520 \mathrm{~g}$, at different positions on each skull and at $1,1.25,1.5,1.75$, and $2 \mathrm{MHz}$ are discussed. They revealed that insertion loss 
through rat skull increases linearly with both body mass and frequency and strongly depends on the position, decreasing from the front to the back and from the midline to the lateral sides. Skull thickness also scales linearly with body mass.

Transcranial focused ultrasound (FUS) in the brain has emerged as a very promising therapeutic tool to replace or complement state-of-the-art therapies [15]. For all in vivo transcranial applications, precise knowledge of the peak negative pressure (PNP) at the focus is key to ensuring both the efficiency and safety of the procedures. Indeed, the acoustic pressure is directly related to the energy deposition in the tissues. This energy deposition must be sufficient to trigger the desired biological effects while avoiding undesired effects at the focus or out-offocus lesions.

To estimate PNP in situ, the first step is to calibrate the ultrasound transducer in a water tank. A second and influential step is correction for ultrasound insertion loss through the skull. The insertion loss results from the addition of several effects contributing to a decrease in the PNP at focus: aberration, reflection, absorption and scattering. The global skull insertion loss strongly depends on the animal species because skull thickness, skull shape and bone structure are unique to each species.

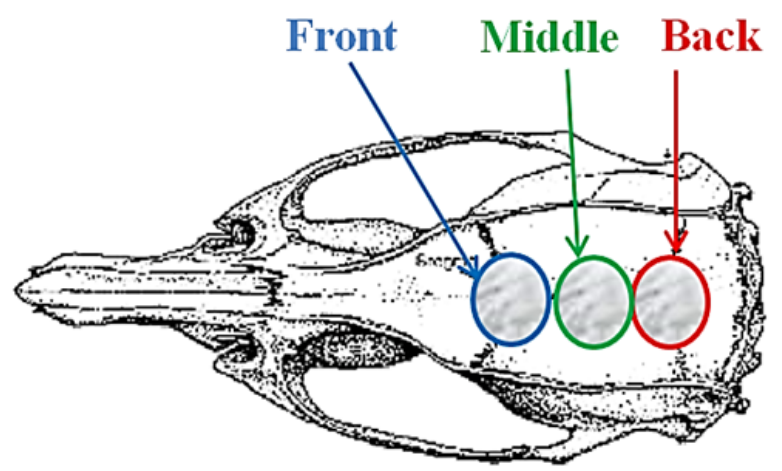

(a)

$$
\begin{aligned}
& \text { (Front) } \mathrm{Y}=1.01 * 10^{-3} \cdot \mathrm{x}+7.63 * 10^{-2} \\
& \mathrm{R}^{2}=0.98 \\
& \text { (Middle) } \mathrm{Y}=9.13 * 10^{-4} \cdot \mathrm{x}+9.48 * 10^{-2} \\
& \mathrm{R}^{2}=0.98 \\
& \text { (Back) } \mathrm{Y}=1.58 * 10^{-3} \cdot \mathrm{x}+2.60 * 10^{-1} \\
& \mathrm{R}^{2}=0.97
\end{aligned}
$$

Fig. 3 Surfaces of the intersection of the ultrasound beam on the rat skull surface for the three different positions (a); skull thickness at three positions as a function of linear regressions are given for each position (b).

In the first experiment, three sets of transmission measurement were done for 10 rat skulls at three different positions along the interhemispheric line-front, middle and back as illustrated in Fig. 3a [15]. The front position corresponds to the striatum, often used in diffusion experiments after US induced blood brain barrier (BBB) opening or to implant tumors for US treatments. The middle position corresponds to the hippocampus, a common target in studies on Alzheimer's disease models. The back position corresponds to the cerebellum, a region for interest. Transmission as a function of frequency and body mass illustrates that the transmission factor decreases linearly with body mass of the rats, at each frequency and at each position (front, middle and back).

The spreading of the US focal spot was measured for a $280 \mathrm{~g}$ rat at the middle position. The width of the focal spot behind the skull was $1.55,1.24$, and $0.95 \mathrm{~mm}$ at 1 , 1.5 and $2 \mathrm{MHz}$, compared with 1.46, 1.23 , and $0.91 \mathrm{~mm}$ without the skull. The length of the focal spot behind the skull was $8.28,5.73$ and $4.95 \mathrm{~mm}$ at $1,1.5$ and $2 \mathrm{MHz}$, compared with $8.15,5.85$, and $4.53 \mathrm{~mm}$ without the skull.

After cutting the skulls, measurements of their thickness obtained with a caliper are given in Fig. 3b. It appears that the skull thickness, at each position, is proportional to the body mass. Skull thicknesses at the front and middle positions are very alike in both their absolute value and their dependency on body mass, unlike thickness at the back, which is higher and increases faster with increasing body mass [15].

\subsection{Through-skull fluorescence imaging of the brain}

In the study [16], through-scalp and through-skull fluorescence imaging of mouse cerebral vasculature without craniotomy, utilizing the intrinsic photoluminescence of single-walled carbon nanotubes (SWCNT) in the 1.3-1.4 $\mu \mathrm{m}$ near-infrared window (NIR-IIa window) was conducted. Reduced photon scattering in this spectral region allows for fluorescence imaging to a depth of $>2 \mathrm{~mm}$ in mouse brain with sub- $10-\mu \mathrm{m}$ resolution. An imaging rate of $\sim 5.3$ frames/sec allows for dynamic recording of blood perfusion in the cerebral vessels with sufficient temporal resolution, providing real-time assessment of a blood flow anomaly in a mouse middle cerebral artery occlusion stroke model.

The 1.3-1.4 $\mu \mathrm{m}$ NIR-IIa window is rationally chosen for in vivo imaging in order to minimize scattering by rejecting photons with wavelengths shorter than $1.3 \mu \mathrm{m}$ while avoiding an increase in light absorption from water vibrational overtone modes above $\sim 1.4 \mu \mathrm{m}$. Dynamic NIR-IIa cerebrovascular imaging with high temporal resolution $(<200 \mathrm{~ms}$ per frame $)$ has also been used to reveal drastically reduced blood flow due to arterial occlusion in an acute stroke model in mice [16].

Live mouse brain imaging was performed noninvasively in vivo at cerebrovascular fluorescence imaging of healthy $\mathrm{C} 57 \mathrm{Bl} / 6$ mice with the SWCNT-IRDye800 conjugates in several spectral 

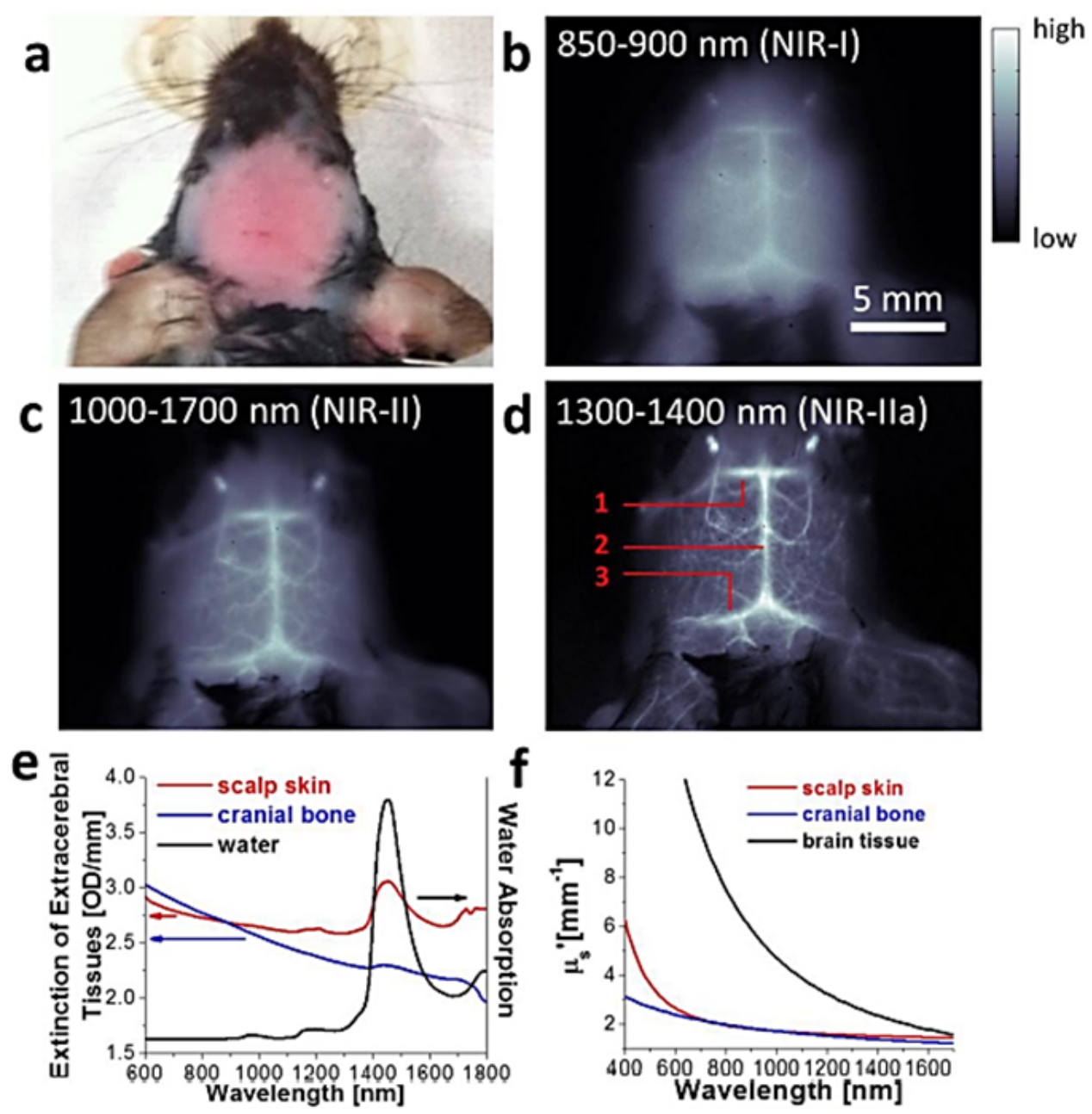

Fig. 4 In vivo mouse brain imaging with SWCNT-IRDye800 in different NIR sub-regions: A C57Bl/6 mouse head with hair removed (a); fluorescence images of the same mouse head in the NIR-I, NIR-II and NIR-IIa regions (b-d); in (d), the inferior cerebral vein, superior sagittal sinus and transverse sinus are labelled 1, 2 and 3, respectively; extinction spectra of scalp (red) and skull (blue) as well as the water absorption spectrum (black) (e); reduced scattering coefficients $\mu_{s}^{\prime}=(1-g) \mu_{s}$ of scalp skin (red), cranial bone (blue) and brain tissue (black) plotted against wavelength, based on the previously reported scattering properties for these tissues (f). Reproduced from Ref. [16]. Copyright 2014, The Authors. Published by Nature Photonics. HHS Public Access, available in PMC 2016, September 16.

windows: NIR-I, NIR-II, and NIR-IIa. A solution of SWCNT-IRDye 800 was injected intravenously through the tail of a mouse. The head of the mouse (with hair shaved off; Fig. 4a) was imaged without craniotomy in the NIR-I $(<900 \mathrm{~nm})$, NIR-II $(1.0-1.7 \mu \mathrm{m})$ and NIR-IIa $(1.3-1.4 \mu \mathrm{m})$ regions, respectively (pixel size $=78 \mu \mathrm{m}$ ), under $808 \mathrm{~nm}$ laser illumination. In contrast to the blurry vasculatures imaged in the NIR-I region (Fig. 4b), much sharper images were obtained by detecting longerwavelength photons (Fig. 4c) and the sharpest and highest resolution images of brain vessels were seen in the NIR-IIa region (Fig. 4d). Similar to the Intralipid phantom imaging case, rejecting the shorter-wavelength $(<1.3 \mu \mathrm{m})$ photons significantly reduced background signals and vessel blurriness caused by scattering, and improved the signal-to-background ratio and imaging quality.
The wavelength dependence of fluorescence imaging of mouse brain can be gleaned by inspecting the extinction spectra of the scalp skin and the cranial bone (the skull), both of which feature a water overtone vibration absorption peak above $\sim 1.4 \mu \mathrm{m}$ and a declining photon scattering profile (Fig. 4e). To examine the effect of scattering, plotted reduced scattering coefficients $\mu_{\text {s }}^{\prime}$ of these tissues versus wavelength $\lambda$ (Fig. 4f), these factors led to drastically improved imaging of mouse cortical vessels underneath the cranial bone in the NIR-IIa region (Fig. 4d versus $4 b$ ).

A new transparent subwindow in the 1.3-1.4 $\mu \mathrm{m}$ wavelength range (the NIR-IIa region) has been created and noninvasive brain imaging in this window by penetrating through the intact skin and skull has been performed at a depth of $>2 \mathrm{~mm}$ with a high spatial resolution of sub-10 $\mu \mathrm{m}$ of cerebral vasculature in an epifluorescence imaging mode [16]. 


\subsection{Multiphoton imaging}

Novel laser spectroscopic techniques for tissues and cells studies are associated with nonlinear spectroscopies, in particular multiphoton (two-photon and three-photon) scanning fluorescence microscopy and optical harmonic generation (second-harmonic generation (SHG) and triple-harmonic generation (THG)) microscopy, which makes it possible to provide in vivo functional imaging [14, 17]. The two-photon technique employs both ballistic and scattered photons at the wavelength of the second harmonic of incident radiation coming to a wide aperture photodetector exactly from the focal area of the excitation beam. A unique advantage of two-photon fluorescence microscopy (TPFM) is the possibility of investigating 3D-distributions of chromophores excited with ultraviolet radiation in thick samples. Multiphoton absorption requires high light intensities in the range $100 \mathrm{MW} / \mathrm{cm}^{2}$ up to $\mathrm{TW} / \mathrm{cm}^{2}$. Most multiphoton microscopes are based on femtosecond laser pulses at $\mathrm{MHz}$ repetition rate with high kilowatt peak power and low mean power in the $\mu \mathrm{W} / \mathrm{mW}$ range [14].

These capabilities stem mainly from the use of NIR femtosecond laser pulses that effectively propagate deeper in biological tissue with less scattering, and localize the two-photon fluorescence excitation generation to the focal spot, and essentially suppress fluorescence out of focus in the background. Brain imaging is one of the most successful fields of application of TPFM inherent from the use of longer excitation wavelengths.

At intensive light interaction with noncentrosymmetrical tissue structures such as collagen, two photons interact simultaneously and generate coherent radiation at exactly half of the excitation wavelength in the forward direction - this is the origin of the second harmonic generation (SHG). There is no light absorption, thus, both photobleaching and photodamage associated with SHG are excluded. The technique enables deep 3Dimaging due to backscattering of light on the second harmonic $[14,17]$.

\subsection{Vibrational energy states excitation and Raman scattering} 4.6.1 MIR and Raman spectroscopies

Mid infrared (MIR) and Raman spectroscopies use light excited vibrational energy states in molecules to get information about the molecular composition, molecular structures and molecular interactions in a sample. In a Raman scattering event, light is inelastically scattered by a molecule, when a small amount of energy is transferred from the photon to the molecule or vice versa. This leads to an excitation of the molecule from usually its lowest vibrational energy level in the electronic ground state to a higher vibrational state. At Raman scattering there is the change of wavelength of the scattered light. The collection of new wavelengths (frequencies) is characteristic to the molecular structure of the scattering medium and differs from the fluorescence spectrum in being much less intense and unrelated to an absorption band of the medium $[14,17]$.

A Raman spectrum represents a molecular fingerprint of the sample and provides quantitative information regarding its chemical composition, as well as a high wavenumber spectrum give a valuable information about tissue water content and its binding ability [17]. Any changes in cells and tissues can lead to significant changes in the Raman spectra. Raman spectroscopy (RS) provides information regarding the inelastic scattering, which occurs when vibrational or rotational energy of target molecules is exchanged with incident probe radiation. Thus, RS is a powerful and useful technique allowing for measuring the chemical composition of various tissue types. Raman spectroscopy and microscopy are widely used in dermatology and skin physiology for in vivo skin analysis [17].

\subsubsection{Terahertz radiation interactions}

The terahertz frequency range lying between the MIR and microwave ranges can be expressed in different terms accounting for that $1 \mathrm{THz} \rightarrow 1 \mathrm{ps} \rightarrow 300 \mu \mathrm{m} \rightarrow$ $33 \mathrm{~cm}^{-1} \rightarrow 4.1 \mathrm{meV} \rightarrow 47.6 \mathrm{~K}$. Terahertz radiation is perspective for molecular spectroscopy and biomedical applications as a less invasive radiation. Due to high water content, tissues have high dispersion and absorption in the terahertz range thus reflection and absorption of this radiation are strong and penetration depth is small [14].

\subsection{Phototherapy 4.7.1 Photodynamic therapy (PDT)}

In clinical medicine, the term PDT is generally defined as the use of a compound or drug (photosensitizer) that has no or minimal effect alone but which, when activated by light, generates one or more reactive chemical species that are able to modify or kill cells and tissues. As historically defined, the PDT reaction should be mediated by oxygen, through the generation of reactive oxygen, most commonly singlet oxygen $\left({ }^{1} \mathrm{O}_{2}\right)$, however, there are photosensitizers that may use oxygen-independent photophysical pathways which could be considered as photodynamic agents also [14].

\subsubsection{Low-level light therapy (LLLT)}

For some diseases, to provide phototherapy no any exogenous phosensitizers are needed and intensity of light could be low, but with a particular wavelength, light dose and light pulse parameters. This is a so-called lowlevel light therapy (LLLT) which uses low intensity visible or NIR light for reduction pain, inflammation and edema; promoting healing of wounds, deeper tissues and nerves, and preventing tissue death. LLLT is still a partly controversial treatment technology which is connected with the lack in understanding of the biochemical mechanisms underlying the positive effects and corresponding difficulties in finding of proper irradiation 
parameters, such as wavelength, fluence, power density, pulse structure, and treatment duration [14].

\subsubsection{Wavelength optimal for transcranial low-level laser stimulation}

One of the challenges in transcranial LLLT is to choose optimal illumination parameters, in particular wavelength. However, there is sparse study on the wavelengths comparison especially on human transcranial LLLT, where authors employed Monte Carlo modeling and visible human head phantom to compute the penetrated photon fluence distribution within cerebral cortex [18].

Transcranial low-level laser stimulation of the brain with light at NIR is a novel form of photobiomodulation, which is well known as LLLT of patients. The illumination wavelength takes a crucial role in deep and proper penetration of the LLLT light into the cerebral tissue with no destructive or uncomfortable effect on the superficial tissue layers.

By innovatively employing the combination of Monte Carlo computation and visible human head phantom, the penetrated light fluence distribution within human head structure originated from the LLLT light stimulation was successfully obtained (see Fig. 5) [18]. This study was the first exploration of optimal wavelength for transcranial LLLT of whole human head in the way of precisely computation of light penetration inside.

Those studies showed that $660 \mathrm{~nm}$ and $810 \mathrm{~nm}$ light could dramatically improve the neurobehavioral performance of mice, when $980 \mathrm{~nm}$ did not repeat any significant positive effect. The $1064 \mathrm{~nm}$ and $980 \mathrm{~nm}$ photons penetrated the superficial layer scalp mostly, which were more likely to induce tissue heating effect rather than photochemical effect, due to absorption of cytochrome $\mathrm{C}$ oxidase (modulate mitochondrial respiration). The accordance between the effectiveness of light penetration in cerebral cortex and the

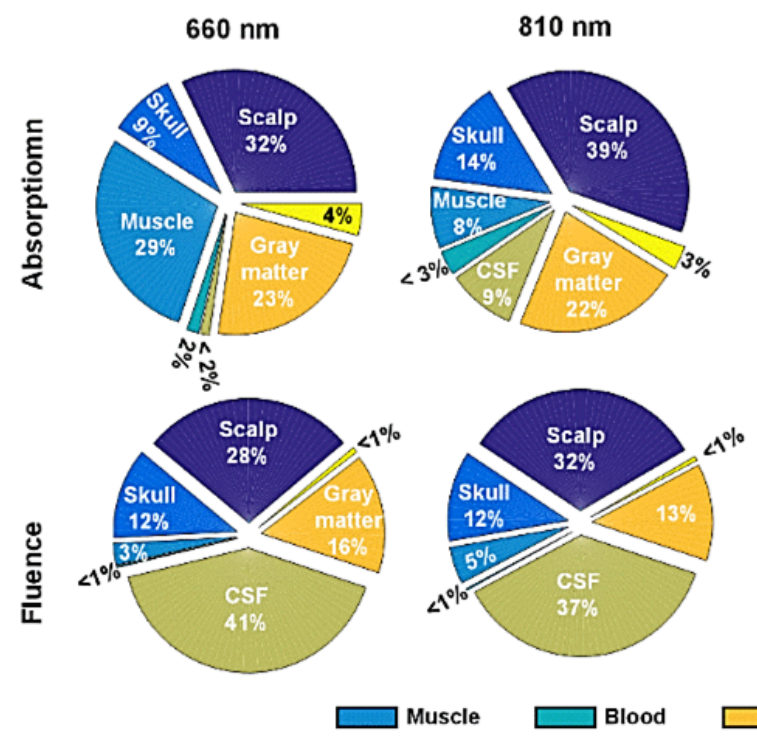

neurobehavioral evaluation consequent upon LLLT was quite surprising and interesting [18].

\subsubsection{Laser light scattering during transcranial rat brain illumination}

Due to the great number of applications of LLLT in Central Nervous System (CNS), the study of light penetration through skull and distribution in the brain becomes extremely important. The aim is to analyze the possibility of precise illumination of deep regions of the rat brain, measure the penetration and distribution of red $(\lambda=660 \mathrm{~nm})$ and NIR $(\lambda=808 \mathrm{~nm})$ diode laser light and compare optical properties of brain structures [19]. The head of the animal (Rattus Novergicus) was epilated and divided by a sagittal cut, $2.3 \mathrm{~mm}$ away from mid plane. This section of rat's head was illuminated with red and NIR lasers in points above three anatomical structures: hippocampus, cerebellum and frontal cortex. A high resolution camera, perpendicularly positioned, was used to obtain images of the brain structures. Profiles of scattered intensities in the laser direction were obtained from the images. There is a peak in the scattered light profile corresponding to the skin layer. The bone layer gives rise to a valley in the profile indicating low scattering coefficient, or frontal scattering. Another peak in the region related to the brain is an indication of high scattering coefficient $\left(\mu_{s}\right)$ for this tissue.

The light scattered by each sample passed through the filter and was captured by a high-resolution camera which was positioned perpendicularly to the laser beam (Fig. 6). The images produced by the scattered light and captured by the camera were analyzed with the software ImageJ 1.44. Intensity profiles, in laser beam direction $(z)$, provide information about tissue optical properties. Profiles of scattered intensities in the laser direction were obtained from the images which are in 8-bits format, so they have 256 gray-levels (gl).

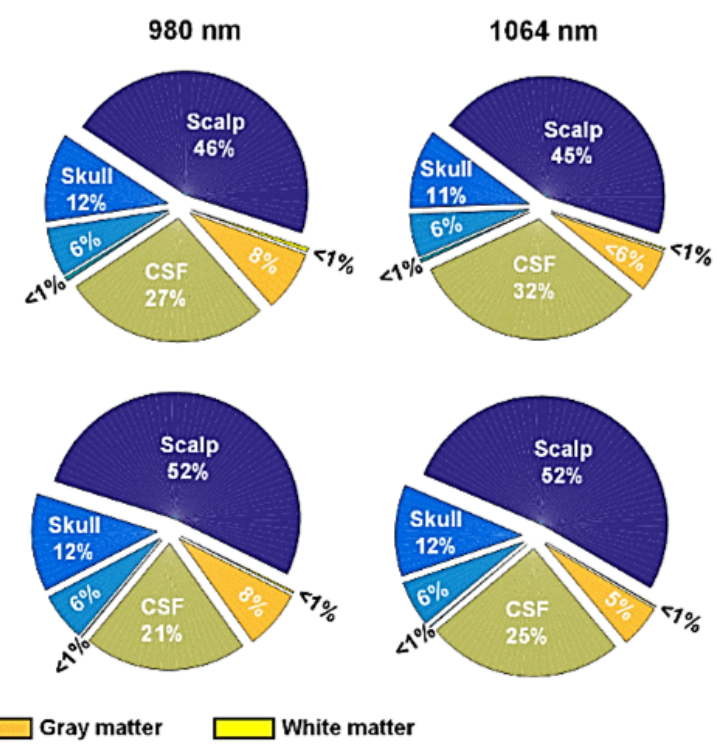

Fig. 5 Pie of photon absorption and fluence distribution in each tissue layers of human head. Reproduced with permission from Ref. [18]. Copyright WILEY-VCH GmbH. 


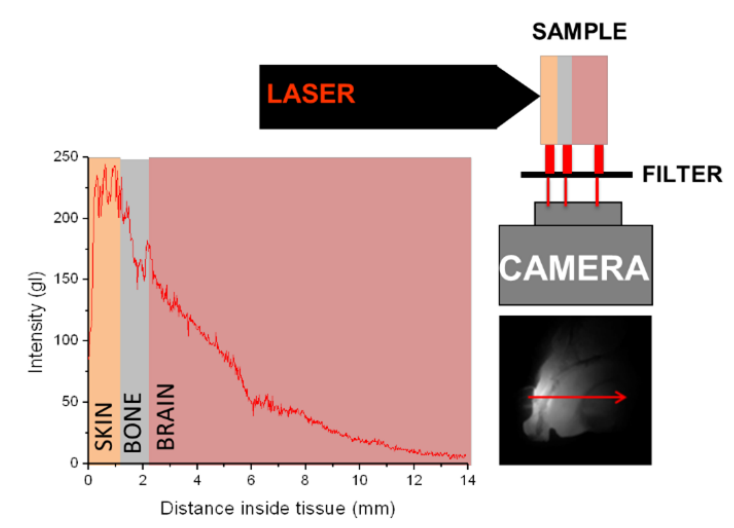

Fig. 6 Setup of the experiment, laser illuminates the sample and scattered light is captured by the camera [19]. Arrow in the image indicates where the light intensity (measured in gray levels in the image - gl) profile shown in the graph. Reproduced from Ref. [19]. Published by SPIE.

Comparing images produced with NIR and red light one is able to notice that NIR penetrates deeper than red light. It suggests that NIR is more indicated to LLLT in inner parts of the brain. The tissue boundaries and the differences of attenuation inside the brain are qualitatively clear in the images. Analyzing intensities profiles of NIR-light images (Fig. 7) one can see that there is a peak in the scattered light profile corresponding to the skin layer. The peak width depends on the thickness of skin in that point, and indicates the high scattering coefficient of skin. The bone layer gives rise to a valley in the profile indicating low scattering coefficient, or frontal scattering. The valley width is proportional to cranial thickness in that point. The following peak in the region related to the brain is an indication of high scattering coefficient $\left(\mu_{s}\right)$ for this tissue.
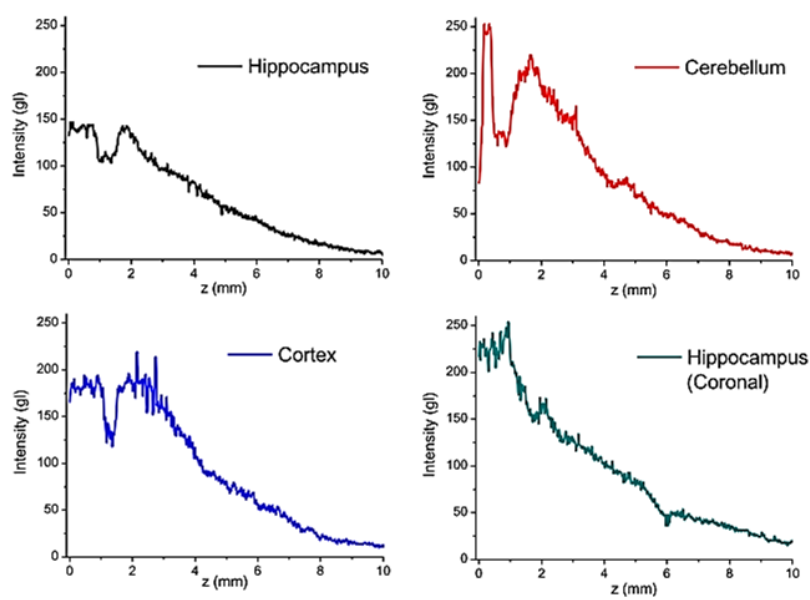

Fig. 7 Profiles of transcranial illumination in different point, NIR $(\lambda=808 \mathrm{~nm})$ illumination, far from the light beam entrance. Reproduced from Ref. [19]. Published by SPIE.

NIR penetrates deeper in a transcranial illumination, skull is not a major attenuator tissue, different parts of brain have different attenuation coefficient; these are useful information to transcranial LLLT. Analyze intensity profiles of images from tissues is a simple and powerful method to study optical properties quantitatively and qualitatively [19].

\subsubsection{Transcranial NIR laser transmission profiles $(800 \mathrm{~nm})$}

Transcranial NIR laser therapy is a promising and novel method to promote neuroprotection and clinical improvement in both acute and chronic neurodegenerative diseases such as acute ischemic stroke, traumatic brain injury, and Alzheimer's disease (AD) patients based upon efficacy in translational animal models [20]. Transcranial NIR laser transmission (NILT) profiles of the skull of 4 different species: mouse, rat, rabbit and human, were measured. For dehydrated skulls from 3 animal species, using a wavelength of $800 \mathrm{~nm}$ and a surface power density of $700 \mathrm{~mW} / \mathrm{cm}^{2}$, NILT decreased from $40.10 \%$ (mouse) to $21.24 \%$ (rat) to $11.36 \%$ (rabbit) as skull thickness measured at bregma increased from $0.44 \mathrm{~mm}$ in mouse to $0.83 \mathrm{~mm}$ in rat and then $2.11 \mathrm{~mm}$ in rabbit. NILT also significantly increased $(p<0.05)$ when animal skulls were hydrated (i.e. compared to dehydrated); but there was no measurable change in thickness due to hydration. In human calvaria, where mean thickness ranged from $7.19 \mathrm{~mm}$ at bregma to $5.91 \mathrm{~mm}$ in the parietal skull, only $4.18 \%$ and $4.24 \%$ of NIR light was transmitted through the skull. There was a slight (9.2-13.4\%) effect of hydration state on NILT of human skulls, but there was a significant positive correlation between NILT and thickness at bregma and parietal skull, in both hydrated and dehydrated states.

A K-1200 dual wavelength (800 $\mathrm{nm}$ and $970 \mathrm{~nm})$ class IV laser (K-Laser LLC, Franklin, TN, USA) with variable power output in the $800 \mathrm{~nm}$ mode was used to reproduce the regimen applied in preclinical therapeutic studies [20]. To determine NILT profiles of the skulls/calvaria as a function of power density three different power densities in the range from 200 to $700 \mathrm{~mW} / \mathrm{cm}^{2}$ were used. The laser light was coupled to a $3 \mathrm{~m}, 600 \mu \mathrm{m}$ fiber (FT600UMT, NA 0.39, Thorlabs Inc, Newton, NJ, USA) which was connected to collimator (F220SMA-780, $780 \mathrm{~nm}, f=11.07 \mathrm{~mm}, \mathrm{NA}=0.26$, Thorlabs). The distance from the lens was adjusted in order to get $11.7 \mathrm{~mm}$ diameter beam. This gave an area of $107.5 \mathrm{~mm}^{2}\left(1.075 \mathrm{~cm}^{2}\right)$ for providing power densities of $200-500-700 \mathrm{~mW} / \mathrm{cm}^{2}$ for rat, rabbit and human samples (Fig. 8). For mouse skulls, an area with diameter of $2.5 \mathrm{~mm}$ was utilized. The laser power was detected using an Integrating Sphere Photodiode Power Sensor (S142C, Thorlabs Inc, Newton, NJ, USA).

Fig. 8 shows an example of a rabbit skull used for NILT analysis. In panel A, the red spotting beam on the surface of the skull at bregma region is shown. In panel $\mathrm{B}$ (left), laser penetration (transmission) through the skull using the red spotting beam and extensive diffusion pattern of laser light measured on the ventral surface of the skull (right) are shown. 


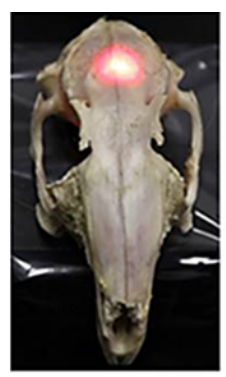

(a)

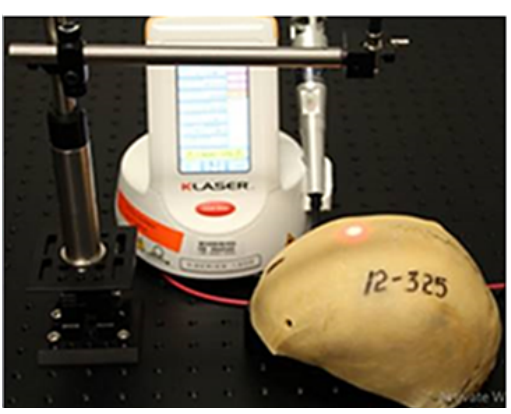

(c)

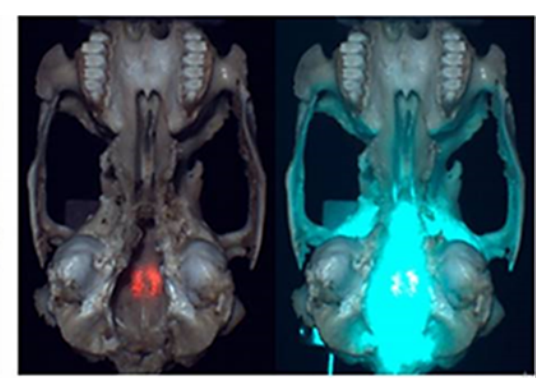

(b)
Fig. 8 NILT: bregma region showing the laser spotting light (a); penetration and scatter ventral surface of the rabbit skull showing the NILT profile and extensive diffusion pattern observed (b); experimental NILT setup for study and treatment at bregma region for human calvaria \#12-325 as an example (c). Images adapted from Ref. [20].

Table 1 Animal skull transmission: comparison of dehydrated-hydrated state (surface power density: $700 \mathrm{~mW} / \mathrm{cm}^{2}$, $800 \mathrm{~nm})[20]$.

\begin{tabular}{|c|c|c|c|c|}
\hline \multirow{2}{*}{$\frac{\text { Species }}{\text { MOUSE }}$} & \multicolumn{2}{|c|}{ Skull thickness (mm) } & \multicolumn{2}{|c|}{ NILT $\left(\mathbf{m W} / \mathrm{cm}^{2}\right)$} \\
\hline & Dry & Wet & Dry & Wet \\
\hline Mouse1 & 0.41 & 0.41 & 305.72 & 441.30 \\
\hline Mouse2 & 0.47 & 0.45 & 269.04 & 423.17 \\
\hline Mouse 3 & 0.46 & 0.47 & 250.70 & 459.44 \\
\hline Mouse4 & 0.40 & 0.39 & 297.57 & 441.30 \\
\hline \multirow[t]{2}{*}{ Mean } & $0.44 \pm 0.01$ & $0.43 \pm 0.01$ & $280.76 \pm 6.35$ & $441.30 \pm 3.70 *$ \\
\hline & & Transmission (\%): & 40.10 & $63.04 *$ \\
\hline RAT & Dry & Wet & Dry & Wet \\
\hline Rat1 & 0.77 & 0.77 & 144.70 & 270.42 \\
\hline Rat2 & 0.90 & 0.89 & 158.01 & 279.73 \\
\hline Rat3 & 0.81 & 0.90 & 145.31 & 261.96 \\
\hline Rat4 & 0.81 & 0.73 & 146.28 & 267.54 \\
\hline \multirow{2}{*}{ Mean } & $0.83 \pm 0.02$ & $0.82 \pm 0.04$ & $148.73 \pm 1.55$ & $269.91 \pm 1.85^{*}$ \\
\hline & & Transmission (\%): & 21.24 & $38.55^{*}$ \\
\hline RABBIT & Dry & Wet & Dry & Wet \\
\hline Rabbit1 & 2.33 & 2.28 & 60.48 & 98.54 \\
\hline Rabbit2 & 2.37 & 2.20 & 60.67 & 91.66 \\
\hline Rabbit3 & 1.60 & 1.71 & 75.44 & 147.31 \\
\hline Rabbit4 & 2.07 & 2.33 & 122.55 & 151.22 \\
\hline Rabbit5 & 2.19 & 2.17 & 78.45 & 138.00 \\
\hline \multirow[t]{2}{*}{ Mean } & $2.11 \pm 0.30$ & $2.14 \pm 0.11$ & $79.52 \pm 5.08$ & $124.55 \pm 5.38 *$ \\
\hline & & Transmission (\%): & 11.36 & $17.79 *$ \\
\hline
\end{tabular}

In Table 1, skull thickness (in mm) for three animal species are presented [20]. As skull thickness increases from $0.44 \mathrm{~mm}$ in mouse to $0.83 \mathrm{~mm}$ in rat to $2.11 \mathrm{~mm}$ in rabbit, NILT $\left(\mathrm{mW} / \mathrm{cm}^{2}\right)$ decreased from $40.10 \%$ in mouse to $11.36 \%$ in rabbit when dehydrated dry skulls were studied. In studies using hydrated and degassed skulls, skulls were submerged in deionized water for $48 \mathrm{~h}$ while under vacuum to remove gases. When hydrated skulls were used for measurement, while there was no measured statistically significant increased NILT (56.6$81.5 \%$ ).

For a surface power density of $700 \mathrm{~mW} / \mathrm{cm}^{2}$ applied to 4 studied species, it was found a greater than $95 \%$ attenuation of NILT through the human skull with approximately $4.5 \%$ of NILT penetrated the human skull for dehydrated human calvaria $(4.18-4.24 \%)$ and this attenuation was not very sensitive to cranial bone hydration state (transmittance of $4.63-4.74 \%$ with hydrated degassed skulls). This is to be compared to NILT of $11.26 \%$ (mean thickness $2.11 \mathrm{~mm}$ ) in rabbits, $21.24 \%$ in rats (mean thickness $0.829 \mathrm{~mm}$ ) and $40.10 \%$ in mice, when measures were done on dehydrate skulls. This data indicates that NILT penetration significantly decreases as skull thickness increases [21, 22].

Table 1, show that both thickness and density vary for each skull measured. Statistical analysis of thickness and density for the entire group of skulls showed that there was no difference $(p>0.05)$ between means, indicating no significant effect of hydration state on skull density or thickness. statistically significant change in thickness $(p>0.05)$, $800 \mathrm{~nm})[20]$ 
NILT penetration for dehydrated and hydrated skulls were not significantly different $(p>0.05)$. Through the results obtained it is found the correlation analysis demonstrates that there is a significant correlation between NILT penetration and skull thickness, whether the skull be dehydrated or hydrated (wet or dry), but there is no correlation between NILT penetration and skull density.

The thickness of the human scalp is 5-6 mm and other major barrier spaces (the periosteum, the dura mater, subdural space and subarachnoid space) are approximately 3-4 $\mathrm{mm}$. Thus, the total barrier distance including skull for NILT is in the range of $14-17 \mathrm{~mm}$. If there is incremental attenuation of NILT due to the additional barriers, even by $50-90 \%$, then the delivered power density on the cortical surface would be estimated to be $2.9-14.5 \mathrm{~mW} / \mathrm{cm}^{2}$.

The human scalp, skull and brain represent a significant barrier that must be overcome if transcranial laser therapy (TLT) efficacy is to be achieved in blinded and randomized clinical trials. Because TLT has been shown to be safe in rodents, rabbits and humans under the specific treatment regimen used in translational studies and clinical trials (trials were safe at $8.3 \mathrm{~mW} / \mathrm{cm}^{2}$ for 2 min per treatment area), TLT neuroprotection should be further developed using an "optimized treatment regimen" that should take into consideration the architecture, anatomy and substantial barriers associated with the human scalp, skull and brain [20-24].

\section{Head tissue optical clearing}

\subsection{Ex vivo and in vivo tissue optical clearing methods}

Every optical imaging technique is limited in its penetration depth by scattering occurring in biological tissues. A possible solution to overcome this problem is to reduce optical inhomogeneities within the sample, i.e. to suppress light scattering. This can be achieved either by using physical methods (such as tissue compression) or by physicochemical methods (such as refractive index matching or the removal of scatterers with the optical clearing agents (OCAs)) basing on tissue optical clearing (OC) protocols [25-31].

It is well known that the major source of scattering in tissues and cell structures is a refractive index mismatch between cell organelles, like mitochondria and cytoplasm, extracellular media and tissue structural components such as collagen and elastin fibers. The tissue scattering properties can be significantly changed due to action of immersion OCAs. Administration of the OCA having a refractive index higher than that of tissue interstitial fluid (ICF) and/or hyperosmotic properties that induces a partial replacement of the ICF by an OCA and hence matching of refractive indices of tissue scatterers and the modified ICF. The matching, correspondingly, causes the decrease of scattering.

The reduction of scattering has proved particularly powerful when performing two photon deep tissue imaging, since it has been demonstrated that scattering causes a drastic reduction in penetration depth (to less than that of the equivalent single-photon fluorescence) while leaving resolution largely unchanged $[17,28]$. On the other hand, the advent of light sheet microscopy has opened the possibility of performing fast volumetric imaging with optical sectioning capability $[27,28]$. This technique drastically reduces acquisition time. Only such a protocol would allow to finally standardize the applications of ex vivo tissue OC methods. For in vivo application the OC efficacy depends on the OCA used as well as on its concentration, leading generally to a more effective $\mathrm{OC}$ as the agent concentration increases. OC methods based on aqueous OCAs were developed to try to preserve endogenous fluorescence and tissue structure. An aqueous solution of glycerol (70-80\% of glycerol) is often used for OC of skin and many other tissues [25-31].

In in vivo brain optical imaging, the underestimation is a recurring pattern in the results and highly depends on the absorption coefficient of the superficial layer [32]. Since fitting procedure, estimates both, $\mu_{a}$ of superficial layer and brain simultaneously, it may be possible to find an underestimation correction factor based on the difference between $\mu_{a}$ superf and $\mu_{a \text { brain }}$ to improve the results. To do this, layered-head model should be used for both forward and inverse problem. This can be investigated further in future studies in the mode of superficial head layer optical clearing.

\subsection{Optical clearing of human dura mater}

The human dura mater (DM) is a protective membrane surrounding the brain. It is a typical fibrous tissue. The bundles of collagen fibrils in the stroma, the layer that forms the main part of the membrane, are arranged in a few rows, interlacing to form a mesh. The mean diameter of collagen fibrils assessed using the electron microscopy data, amounts to $100 \pm 5 \mathrm{~nm}$. The thickness of DM varies from 0.3 to $0.9 \mathrm{~mm}$ depending on the age. At present, different hyperosmotic liquids are used as the OCAs, e.g., the solutions of glycerol, propylene glycol, some radiopaque substances (Trazograph, Verographin, Omnipaque), etc $[25,26,30,31]$. For their clinical application, one should consider both their biocompatibility and their diffusion rate in the tissues. One of the promising candidates for the role of OCA is the aqueous glucose solution that is allowed for clinical use, possesses sufficiently high refractive index [30].

\subsection{Optical clearing of cranial bone}

Both transmittance and reflectance spectra of human and porcine cranial bone samples were measured in vitro $[33,34]$. For estimation of absorption and reduced scattering coefficients of the bone, the inverse addingdoubling method was used. The decrease of reflectance of the samples under action of the immersion agents was demonstrated. The experiments have shown that administration of the immersion liquids allows for effective controlling of tissue optical properties that makes bone more transparent, thereby increasing the 
ability of light penetration through the tissue. The possibility of selective translucence of cranial bone can be very useful in developing techniques of brain functional imaging.

At microstructural length scales, cortical bone is organized into 200-300 $\mu \mathrm{m}$ diameter secondary osteons, which are composed of large vascular channels (50$90 \mu \mathrm{m}$ diameter) surrounded by circumferential lamellar rings (3-7 $\mu \mathrm{m}$ thick), with the so-called "cement lines" at the outer boundary. At the nanostructural level, the lamellae are composed of organic type-I mineralized collagen fibers (up to $15 \mu \mathrm{m}$ in length, $50-70 \mathrm{~nm}$ in diameter, and formed by regular arrangement of subnanostructural collagen molecules) bound and impregnated with inorganic carbonated apatite nanocrystals (about $30 \mathrm{~nm}$ in length and width, 2-3 nm in thickness) [34].

The measurements of bone reflectance have been performed in the spectral range 450-1000 nm using a commercially available optical multichannel spectrometer LESA5 ("BioSpec," Moscow, Russia) with a fiber-optical probe. Reflectance spectra of cranial porcine bone during the action of $100 \%$-propylene glycol as an OCA were measured. In the spectra, three spectral bands corresponding to blood absorption of the $\alpha$-band with maximum at $537 \mathrm{~nm}$, the $\beta$-band with maximum at $568 \mathrm{~nm}$ and the long-wavelength wing of the Soret band of oxyhemoglobin with maximum at $415 \mathrm{~nm}$ were found. The action of the OCA produces the decrease of the reflectance of the bone tissue in the entire studied spectral range that indicates that bone becomes more transparent due to decreasing of light scattering. Before and during

a

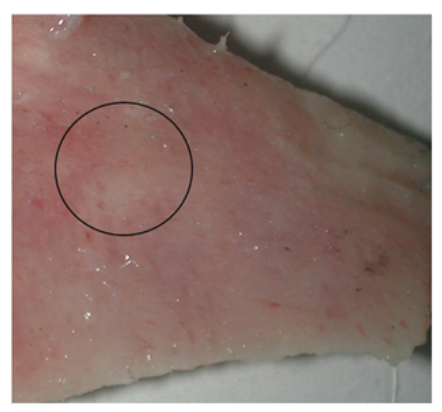

c

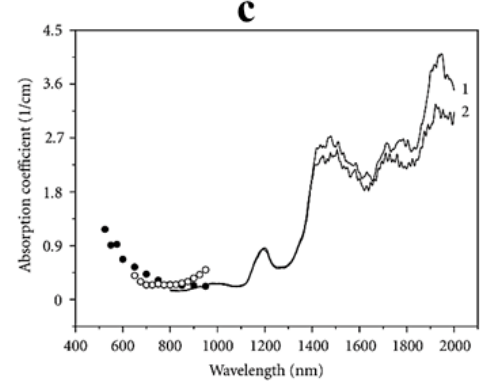

OC under action of $100 \%$ propylene glycol images of cranial porcine bone were captured by a digital camera. In the image, the printed on the paper "cross" behind bone sample is well seen after OC (Figs. 9a and 9b).

The optical properties of the human cranial bone samples in vitro before and after action of glycerol have been determined over the wavelength range 800 $2000 \mathrm{~nm}$ using the integrating sphere technique and the inverse adding-doubling (IAD) software [33, 34]. The total transmittance and diffuse reflectance measurements of the samples have been performed using the commercially available spectrophotometer CARY-2415 ("Varian", Australia). Inner diameter of the sphere is 100 $\mathrm{mm}$, size of the entrance port is $20 \times 20 \mathrm{~mm}$, and diameter of the exit port is $16 \mathrm{~mm}$. The diameter of incident light beam on the tissue sample is $3 \mathrm{~mm}$. Scan rate is $2 \mathrm{~nm} / \mathrm{s}$. The measurements were carried out at room temperature about $20{ }^{\circ} \mathrm{C}$. An important advantage of the IAD method when applied to tissues is a possibility of rapidly obtaining iterative solutions. This method allows one to determine the absorption $\left(\mu_{a}\right)$ and the reduced scattering coefficients $\left(\mu_{s}^{\prime}=(1-g) \mu_{s}\right)$ of a tissue from the measured values of the total transmittance and the diffuse reflectance. In these calculations, the anisotropy factor has been fixed as $g=0.9$, since this value is typical for the tissue in the visible and NIR spectral ranges. Figs. 9c and 9d demonstrate kinetics of optical properties of human cranial bone at administration of glycerol in spectral range 800-2000 $\mathrm{nm}$. Using these data, calculation of the optical penetration depth has been performed.
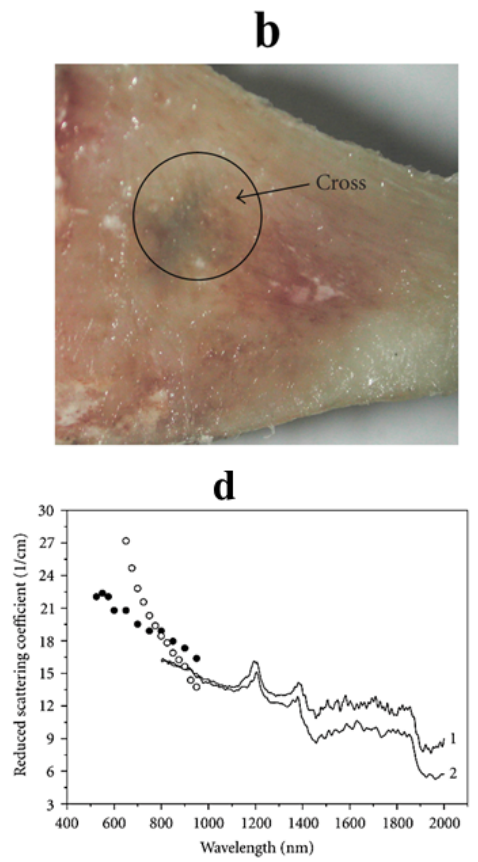

Fig. 9 Optical clearing of cranial bone samples: cranial porcine bone sample before (a) and after (b) OC using $100 \%$ propylene glycol during $24 \mathrm{~h}$, thickness of the sample was $1.5 \pm 0.2 \mathrm{~mm}$; the absorption spectra of the human cranial bone sample measured before (1) and after administration of glycerol (2) during one hour (c); the reduced scattering coefficient spectra of the sample measured before (1) and after administration of glycerol (2) during one hour (d); black circles correspond to data presented in Ref. [35] and open circles correspond to data presented in Ref. [36] without OC. Images adapted from Ref. [34]. 
The penetration depth is maximal in the spectral ranges 800-900 nm and 1000-1100 nm, where the optical radiation penetrates up to depths of $0.32-0.37 \mathrm{~cm}$ (light attenuation of 2.7-fold) in intact cranial bone. During an hour after administration of glycerol, the optical penetration depth increases by $5 \%$ in these two spectral ranges. In the spectral range $1400-2000 \mathrm{~nm}$, the penetration depth increases more than $20 \%[33,34]$.

Recently, Zhu's group invented an innovative skull optical clearing solution (SOCS) [37]. With this technique, the skull can be transparent within $25 \mathrm{~min}$, and the high scattering restriction of the skull for cortical imaging in vivo could be overcome, and the cortical blood flow with high resolution can be obtained. The purpose of this study is to quantitatively evaluate the performance of SOCS systematically. The improvement of divergence of beam spot and resolution were investigated before and after the treatment of SOCS on mouse skull. Transmittance combined with Monte Carlo (MC) simulation was used to evaluate the changes in scattering coefficient after applying SOCS. Before measurements, the fresh mouse skulls were immersed and kept at $4{ }^{\circ} \mathrm{C}$ in PBS. An optical fiber spectrometer (USB 4000, Ocean Optics, USA) was used to measure the transmittance of the mouse skull. Fig. 10 shows the schematics of measurement system. A light source (HL-2000-CAL) coupled with an optical fiber (QP600-2-VIS-NIR) was used to illuminate on the region of interest of the skull, and another similar optical fiber coupled with spectrometer system was used to collect the transmission light in the spectral range of 400-900 nm.
By applying this equipment, the transmittance spectrum of skull can be obtained before and after SOCS application. The collimated transmittance $(T)$ was calculated as

$$
T_{c}=\left\{\left(S_{\text {sample }}-S_{\text {dark }}\right) /\left(S_{\text {empty }}-S_{\text {dark }}\right)\right\} \times 100 \%,
$$

where $S_{\text {sample, }} S_{\text {empty, }}$ and $S_{\text {dark }}$ are the light intensity spectra in the state of placing sample, removing sample and dark noise, respectively.

In order to obtain the scattering coefficient of skull, the relation between coefficient and collimation transmittance was established by using MC simulation. Here, the skull was assumed as a uniform media, and the parameters of skull were set as follows, i.e., the absorption coefficient, mean refractive index and thickness of skull were set as follows, $\mu_{a}=0.5 \mathrm{~cm}^{-1}$, $n=1.555, d=300 \mu \mathrm{m}, g=0.911-0.958$, respectively, and the scattering coefficient, $\mu_{s}=30-250 \mathrm{~cm}^{-1}$ due to optical clearing. The above parameters were thought to be unchangeable except $\mu_{s}$ and $g$. Therefore, it would obtain the number of transmittance photons in the area of detect probe by using MC simulation, which would change with scattering coefficient of skull. Further, could deduce the coefficient based on the measurement of collimation transmittance of skull. Fig. 10A shows the measured transmittance in the wavelength range of $500-900 \mathrm{~nm}$ before or after treatment of SOCS on skull, which increases as the wavelength extending.
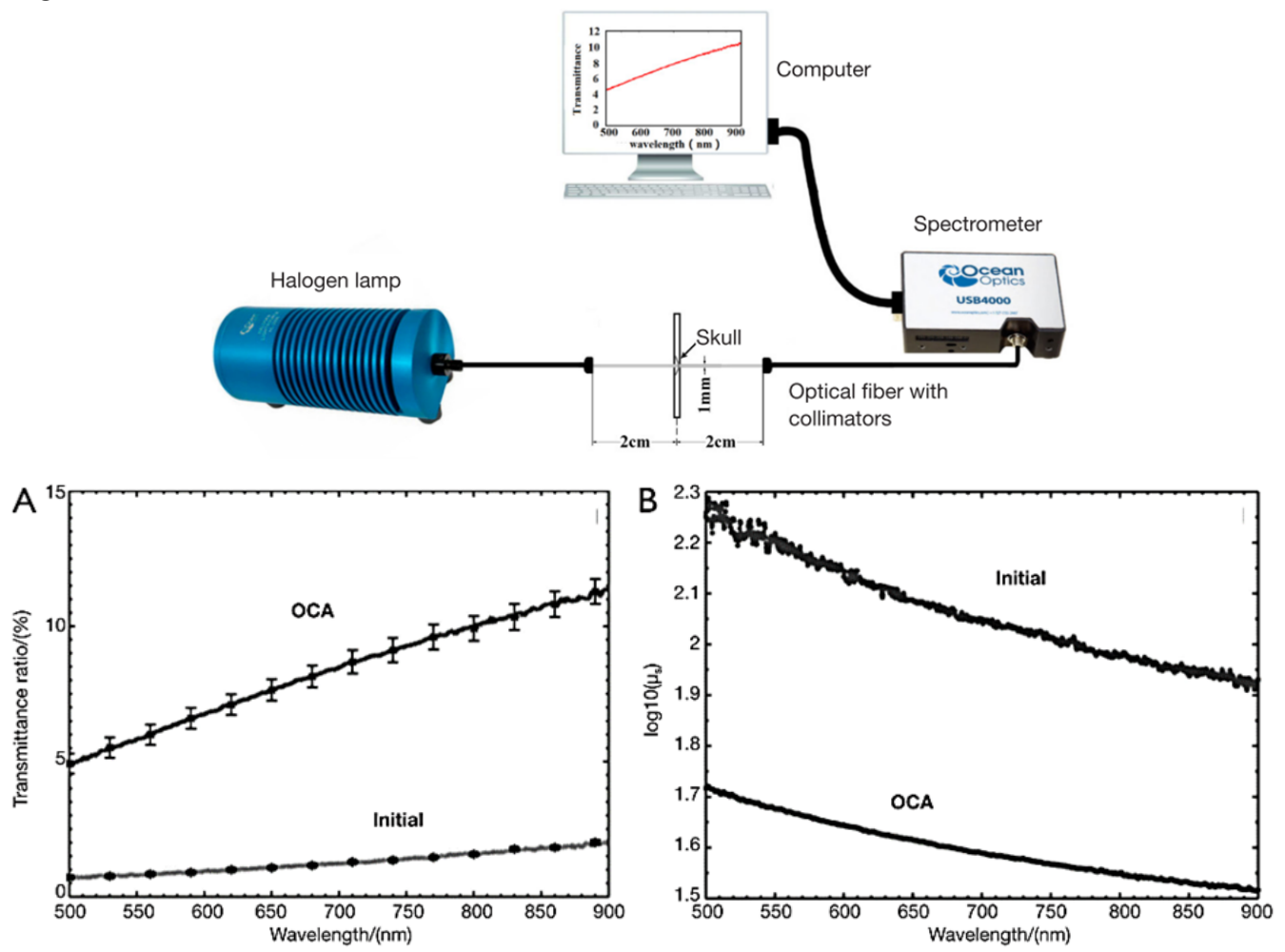

Fig. 10 Schematics of optical fiber spectrometer system for measuring collimation transmittance of the skull (top); the transmittance spectra under two states, before and after applying SOCS-OCA (A); the relations between $\log _{10}\left(\mu_{s}\right)$ and wavelength by combining the results of transmittance and MC simulation (B). Reproduced from Ref. [37] published under CC BY-NC-ND 4.0 license. 
For the intact skull, the transmittance of skull is relatively low at $0.5 \%$ to $2 \%$. After applying SOCS, the collimation transmittance raises with an overall upward trend from $5 \%$ to $12 \%$ in the same range of wavelength. The results showed that the measured beam bandwidth reduced from $5.2 \pm 0.3$ to $2.0 \pm 0.2 \mathrm{~mm}$; and the scattering coefficient decreased nearly three-fold after the treatment of SOCS on skull [37].

\subsection{Multiphoton tomography at optical clearing}

MPT is an efficient tool for noninvasive in vivo skin examination. Based on two- and three-photon excitation of fluorophores, this method allows one to investigate the 3D distribution of fluorophores in thick biological samples. The SHG signal is emitted by collagen type I molecules. As a rule, the wavelengths longer than the wavelengths of tissue autofluorescence excitation are used in MPT. The main drawback of MPT lies in the fact that strong scattering in biological tissues critically decreases the penetration depth of the focused laser radiation to the depth that is even smaller than for the equivalent one-photon fluorescence microscopy. That is why the application of OCAs in MPT studies of tissues can significantly enhance the potential of this nonlinear optical imaging method [38]. Despite the fact that both glycerol and Omnipaque ${ }^{\mathrm{TM}} 300$ provided significant OC, glycerol was more efficient as an OCA, although it caused a pronounced compression of skin tissues and some changes in cell morphology. No such effects were observed for iso-osmolar Omnipaque ${ }^{\mathrm{TM}} 300$ solution, and that is why suggest it as a safe and fast acting OCA for clinical tests [38]. OC with glucose was also 3 times faster than with glycerol. In addition, a higher degree of tissue dehydration in the case of glycerol could also be the reason for a lower SHG efficiency for a longer action of an OCA.

An easy-handling and safe skull optical clearing window (SOCW) for in vivo imaging of the cortical structures at synaptic resolution with two-photon microscopy enables one to repeatedly image the dendritic protrusions, microglia processes and blood capillaries in the superficial layers of the cortex; and applied this approach to monitor the plasticity of dendritic protrusions in critical periods and to visualize the changes in dendrites and microglia upon laser injury [39]. First, observed microglia in vivo 0 and 2 days after craniotomy and after $\mathrm{OC}$, respectively. Found that microglia remained at the same position when viewed through the SOCW, which indicates that the SOCW technique does not affect the microenvironment. In contrast, the distribution of microglia seen through the open-skull glass window was completely different. The craniotomy evidently induced the movement of microglia (see Fig. 11). a

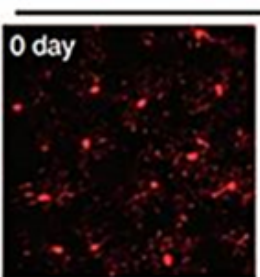

Distribution of microglia

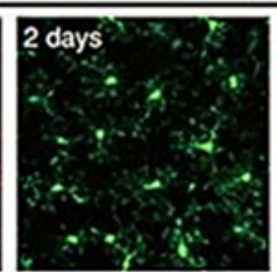

C
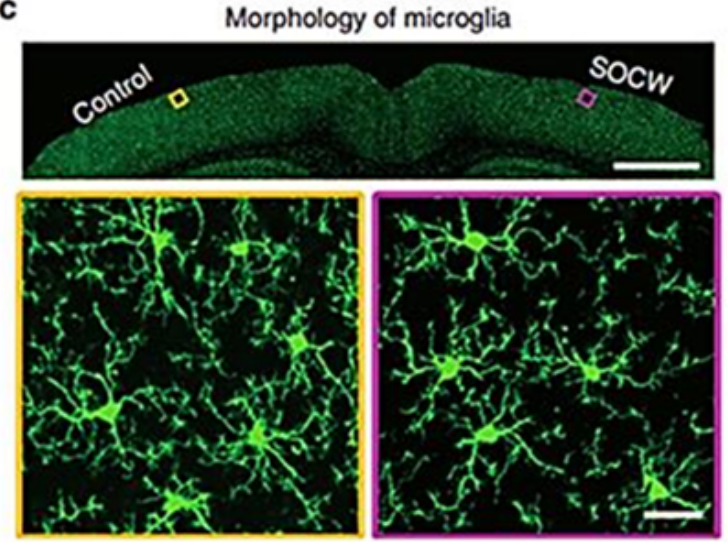

b
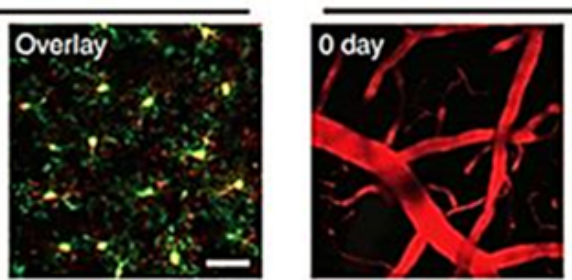

Distribution of blood vessels

d
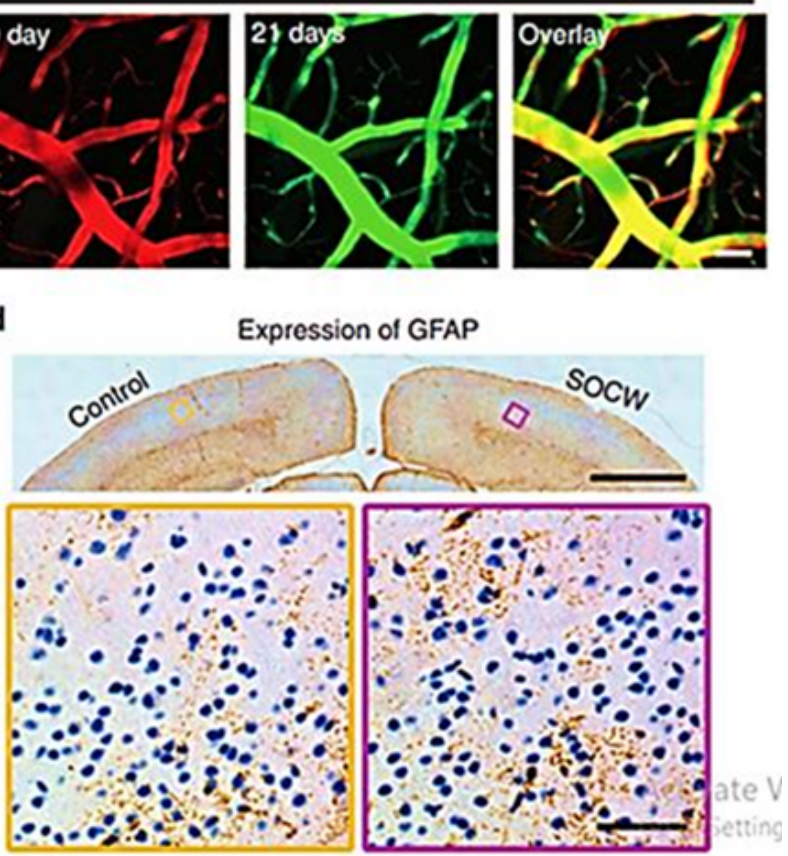

Fig. 11 Safety assessment of the SOCW technique: distribution of microglia through the SOCW, microglia remain at the same position (P28, $\mathrm{n}=4$ mice), scale bar $=25 \mu \mathrm{m}(\mathrm{a})$; maximum projections ( $z$-axis) across $10-40 \mu \mathrm{m}$ of FITC-dextranfilled cerebral vasculature under the SOCW obtained $0(\mathrm{P} 28, \mathrm{n}=4$ mice) and 21 days after the treatment, the cerebrovascular morphology remains nearly unchanged 21 days after forming the SOCW, scale bar $=25 \mu \mathrm{m}$ (b); histological images of microglia in the case of the SOCW; microglia in both the treated and control sides appear normal $(\mathrm{P} 30, \mathrm{n}=3 \mathrm{mice})$, scale bar $=1 \mathrm{~mm}$ (above) and $25 \mu \mathrm{m}$ (below) (c); GFAP expression under the SOCW, the treated and control hemispheres show similar levels of GFAP expression (P38, $\mathrm{n}=3$ mice), scale bar $=1 \mathrm{~mm}($ above) and $50 \mu \mathrm{m}$ (below) (d). Images adapted from Ref. [39]. 


\section{Measurements of optical properties of head tissues}

\subsection{Tissue optical transparency windows}

Authors of Ref. [4] have obtained optical collimated transmittance spectra in ex vivo studies of the healthy rat brain cortex, cranial bone and skin samples with thicknesses of $1,0.9$, and $1 \mathrm{~mm}$, respectively. All the spectra clearly exhibit the dominating water absorption bands peaked at $\sim 1450,1950 \mathrm{~nm}$ and above $\sim 2200 \mathrm{~nm}$ (Fig. 12a). Four tissue transparency windows: $\sim 700$ to $1000 \mathrm{~nm}$ (NIR-I), $\sim 1000$ to $1350 \mathrm{~nm}$ (NIR-II), $\sim 1550$ to $1870 \mathrm{~nm}$ (NIR-III or SWIR) and 2100 to $2300 \mathrm{~nm}$ (NIR-IV or SWIR-II) are recognized. SWIR window demonstrates the highest light penetration (the lowest the attenuation coefficient $\left(\mu_{t}\right)$ ) for all the as-prepared rat brain cortex, cranial bone and skin samples, the transmittance is lower in NIR-I and lowest in NIR-II and SWIR windows (Fig. 12b).

All the presented data revealed strong increase in the transmittance with wavelength in NIR together with the trend that the gray matter is more transparent than the white.

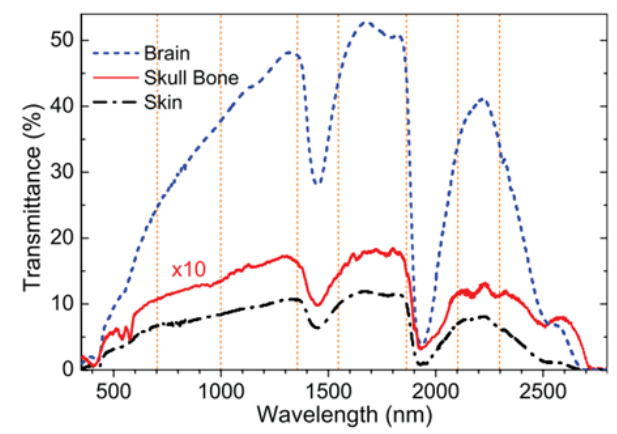

(a)

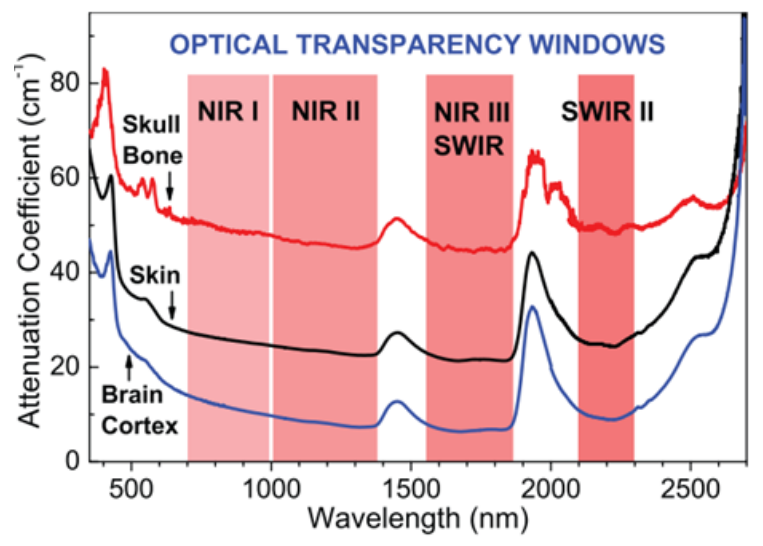

(b)

Fig. 12 Spectra of the collimated transmittance for rat head tissues: brain cortex, cranial bone and skin (a) and corresponding spectra of the attenuation coefficient (b) [4]. Four optical transparency windows are indicated $[4,5]$. Reproduced with permission from Ref. [4]. Copyright WILEY-VCH GmbH.
In case of SWIR window (at $\sim 1700 \mathrm{~nm}$ ), $\mu_{t}$ of the human cortex was determined to be 20 to $35 \mathrm{~cm}^{-1}$. Water absorption significantly determines the permeability of tissues; it has peaks around 970, 1180, 1450, 1775, 1930, and $1975 \mathrm{~nm}$. The peak at $\sim 1450 \mathrm{~nm}$ is referred to the first overtone of $\mathrm{O}-\mathrm{H}$ stretching. Whole blood intensely absorbs light in the visible, where the characteristic hemoglobin bands peaked at $\sim 420$ and $\sim 550 \mathrm{~nm}$. There are some disparities between soft head tissues and bone: the absorption spectra of skin and brain cortex exhibited a single absorption peak at $550 \mathrm{~nm}$ related to deoxyhemoglobin $(\mathrm{Hb})$, while the double-peak absorption at 540 and $575 \mathrm{~nm}$ from oxy-hemoglobin $\left(\mathrm{HbO}_{2}\right)$ is detected for skull bone. Lipids are one of the important constituents of biological tissues, exhibiting major absorption peaks at 920,1040, 1210, 1430, 1730, 1760, and 1900 to $2600 \mathrm{~nm}$. Higher lipid content results in stronger bone absorption in SWIR range if comparing with the soft tissues. Proteins also manifest weak bands at around 1420 to 1600 and 800 to $1100 \mathrm{~nm}$ and the skull bone spectra display more prominent broad collagen bands at 1945, 2050, 2170, 2300, and $2350 \mathrm{~nm}$ (see Fig. 13).

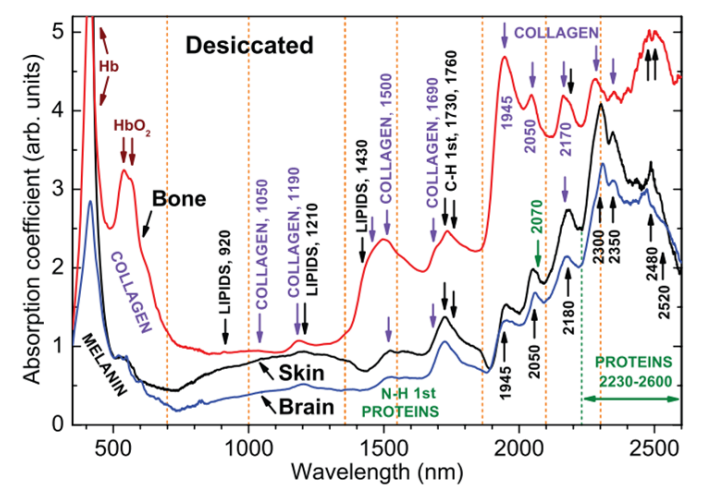

Fig. 13 Spectral dependences of the absorption coefficient for the brain (blue), skull bone (red) and skin (black) samples after the desiccation. The major bands of the generic tissue components are marked. Reproduced with permission from Ref. [4]. Copyright WILEY-VCH $\mathrm{GmbH}$.

In the study [4], $\mu_{t}(\lambda)$ was calculated for rat brain cortex, cranial bone and scalp skin in the spectral range from 350 to $2700 \mathrm{~nm}$, using the collimated light transmittance described by the Beer-Lambert law:

$$
T_{c}(\lambda)=\frac{I_{c}}{I_{o}}=e^{-\mu_{t}(\lambda) d},
$$

where $d$ is the light path length (equal to tissue sample thickness), considering that the total attenuation coefficient of a tissue layer is the sum of the absorption $\left(\mu_{a}\right)$ and scattering $\left(\mu_{s}\right)$ coefficients:

$$
\mu_{t}=\mu_{a}+\mu_{s} .
$$

In the study [4] for rat head tissues, after passing the head skin of $\sim 1 \mathrm{~mm}$ thickness, light is attenuated 
$\sim 10$ times. While propagating through the $0.9 \mathrm{~mm}$ thick skull bone, as a result of strong scattering and absorption, light is attenuated $\sim 50$ to 80 times (depending on the spectral window) more than in the skin. Finally, when light penetrates into the brain cortex tissue, its intensity decreases by 2 to 2.8 times per $1 \mathrm{~mm}$ of the path, and the values of light intensity after all three types of head tissues are $0.11 \%, 0.088 \%, 0.042 \%$, and $0.039 \%$ of the initial incident light in SWIR, NIR-II, SWIRII and NIR-I windows, respectively [4].

While the authors of Ref. [40] were determined optical properties of ex vivo mouse skull bone in the 455 to $705 \mathrm{~nm}$ spectral range. Similar reduced scattering coefficients were published by Firbank et al. [36] for the pig skull. However, results of Ref. [40] show large differences in absorption coefficients measured at 455 to $705 \mathrm{~nm}$, with relatively strong discrepancy with the data at 650 to $705 \mathrm{~nm}$. A source of potential errors in the IAD method is the assumptions made on the material homogeneity, thickness and refractive index.

As expected, the absorption coefficient for fresh skull presents blood absorption peaks characteristic to oxyhemoglobin absorption in the visible range with two maxima, respectively, at 537 and $568 \mathrm{~nm}$. These two peaks strongly diminished after using $\mathrm{H}_{2} \mathrm{O}_{2}$. The average reduced scattering coefficients for the first group are shown in Fig. 14d. Reduced scattering coefficients vary from $2.79 \pm 0.26 \mathrm{~mm}^{-1}$ at $455 \mathrm{~nm}$ to $2.29 \pm 0.12 \mathrm{~mm}^{-1}$ at $705 \mathrm{~nm}$ before $\mathrm{H}_{2} \mathrm{O}_{2}$ and varied from $4.17 \pm 0.34 \mathrm{~mm}^{-1}$ at $405 \mathrm{~nm}$ to $3.25 \pm 0.24 \mathrm{~mm}^{-1}$ at $705 \mathrm{~nm}$ after $\mathrm{H}_{2} \mathrm{O}_{2}$ [40].

\subsection{Optical properties of brain tissues at the different stages of glioma development in rats}

The penetration of light in the visible/NIR into healthy brain is well characterized but not to gliomas [6]. Gliomas are one of the most common types of primary brain tumors and the leading cause of death by brain diseases in both children and adults. Further to this, tumors are graded (I, II, III, and IV) according to their anticipated biological behavior, from the least malignant to the most malignant: grade I has benign cytological features, grade II is characterized by moderate cellularity without anaplasia or mitotic activity, grade III has cellularity, anaplasia, mitoses, and grade IV is same as grade III plus microvascular proliferation and necrosis. Therefore, the study [6] has aimed to measure absorption and scattering coefficients and anisotropy factor of brain tissues in healthy rats and rats with a C6 model glioblastoma $(7,10$, and 30 days) in a wide spectral range from 350 to $1800 \mathrm{~nm}$ (Fig. 15). The structure and physiological properties of C6-glioma is multiforme, the most common and aggressive form among all brain tumors (WHO grade IV). The C6-glioma cell line is morphologically similar to human glioblastoma.

The inverse Monte Carlo (IMC) method was used for determination of the absorption $\left(\mu_{\mathrm{a}}\right)$ and the scattering $\left(\mu_{\mathrm{s}}\right)$ coefficients, and scattering anisotropy factor $(g)$ of a tissue from the measured values of the total and collimated transmittance and the diffuse reflectance [6].
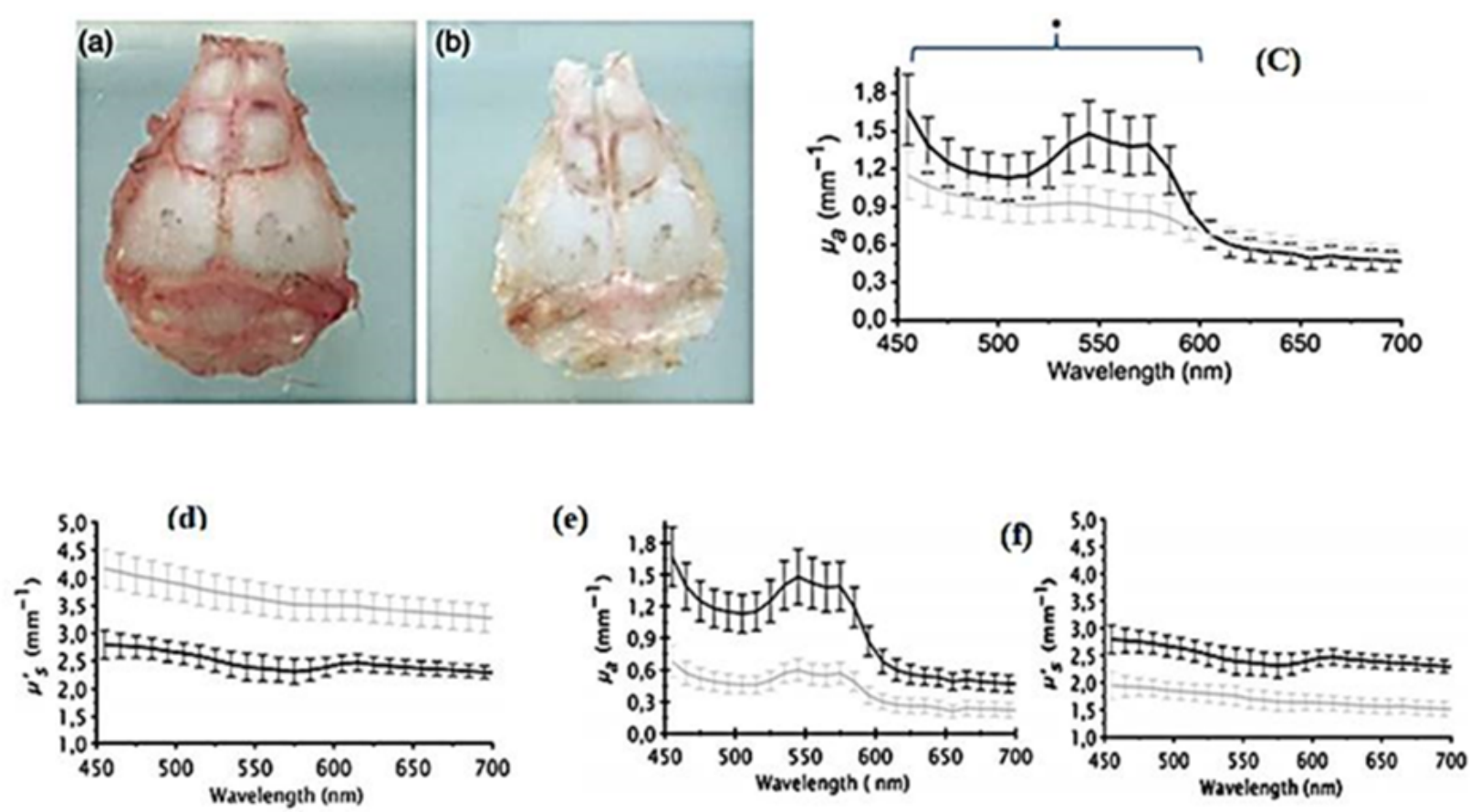

Fig. 14 The mouse skull bone sample before (a) and after (b) treatment in $\mathrm{H}_{2} \mathrm{O}_{2}$ for 30 min; absorption coefficient of skull measured before (black curve) and after treatment with $\mathrm{H}_{2} \mathrm{O}_{2}$ (grey curve) (c); reduced scattering coefficient spectra of skull measured before (black curve) and after treatment with $\mathrm{H}_{2} \mathrm{O}_{2}$ (grey curve) (d); influence of age on the optical properties of the skull for 4-month-old (black curves) versus 4-week-old (grey curves) mice (e, f). Reproduced from Ref. [40]. Published by SPIE. 


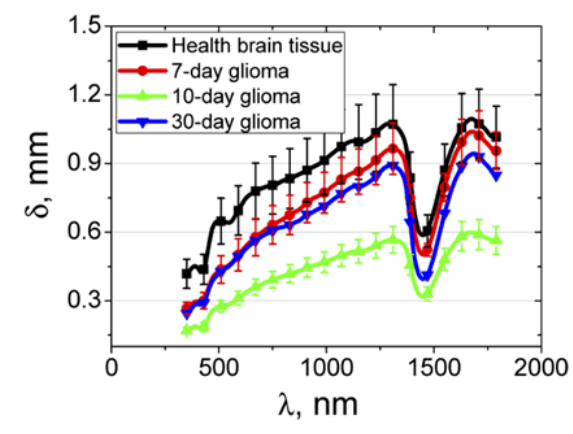

(a)

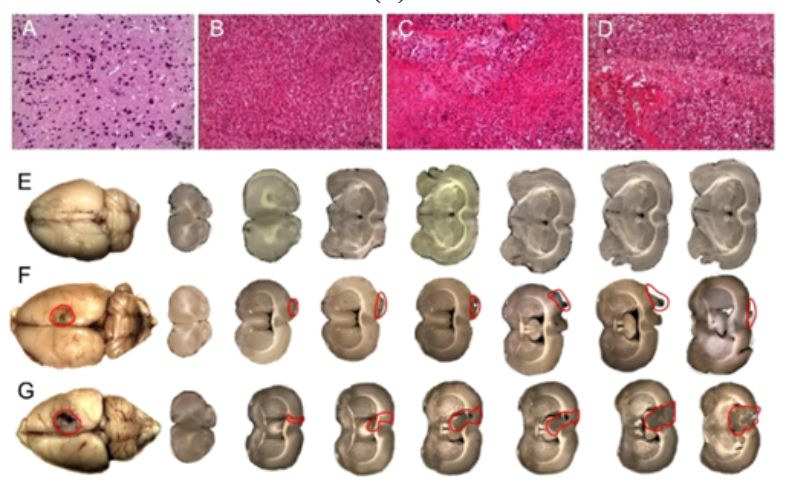

(b)

Fig. 15 Glioma progression in rats [6]: the light penetration depth into the healthy brain tissues and 7-10-30 days after glioma implantation, the solid line corresponds to the mean experimental values and bars to the standard deviation (a); morphological and behavior signs of glioma progression (b): A-D - histological imaging of healthy brain and 7-10-30 days after glioma implantation; E-G - digital images of healthy brain and 10-30 days after glioma implantation. Reproduced from Ref. [6]. Published by OSA.

The IMC method included two steps. In the first step, the experimental data (the total and collimated transmittance and the diffuse reflectance) were processed by the IAD method. In the second step the resulting values of $\mu_{a}, \mu_{s}$ and $g$ were refined using the IMC method. The C6-glioma growing is assisted by edema that, apparently, decreases the volume fraction of the scatterers from $\sim 0.2$ for normal tissue to $\sim 0.15$, and consequently, decreases the scattering coefficient. Increase of the scattering properties observed after 10day glioma development could be due to increase of the tumor vascularization, and therefore appearance of the large-size scatterers (blood erythrocytes, vessel walls, etc.). Apparently, during the development of hemorrhages, erythrocytes from damaged vessels penetrate into surrounding tissues and ISF. In this case, cell membranes and parts of damaged cells are scatterers, and hemoglobin flowing out of erythrocytes causes the refractive index to be matched between the scatterers and ISF, which, accordingly, reduces scattering. The behavior of scattering in the intermediate stages of glioma development can be caused by gradual tissue damage during the glioma growing into surrounding tissues (grey and white matter) [6]. Using spectral data for $\mu_{a}(\lambda), \mu_{s}(\lambda)$ and $g(\lambda)$, tissue penetration depth $\delta(\lambda)$ in diffusion approximation can be calculated (Fig. 15a):

$$
\frac{1}{\delta(\lambda)}=\sqrt{3 \mu_{a}\left[\mu_{a}+\mu_{s}(1-g)\right]}=\sqrt{3 \mu_{a}\left(\mu_{a}+\mu_{s}^{\prime}\right)} .
$$

\subsection{Evaluating optical properties of brain in vivo}

In another study, diffuse reflectance spectroscopy using fiber optic probe, which is one of most promising technique for evaluating optical properties of biological tissue in vivo, was applied to brain tissues [41]. The reduced scattering coefficient $\mu_{s}^{\prime}$, the absorption coefficient $\mu_{a}$, and tissue oxygen saturation $\mathrm{StO}_{2}$ of in vivo rat brain tissue were determined using a fiber probe with two source-collector geometries. In vivo recordings of diffuse reflectance spectra and the electrophysiological signals for exposed brain of rats during the cortical spreading depression (CSD) evoked by the topical application of $\mathrm{KCl}$ were performed. The time courses of $\mu_{a}$ in the range from 500 to $584 \mathrm{~nm}$ and $\mathrm{StO}_{2}$ indicated the hemodynamic change in cerebral cortex. Time courses of $\mu_{s}^{\prime}$ are well correlated with those of $\mu_{a}$ in the range from 530 to $570 \mathrm{~nm}$, which also reflect the scattering by red blood cells.

Fig. 16a shows the schematic illustration of measuring area and stimulating sites on the rat head. A longitudinal incision about $20 \mathrm{~mm}$ was made along the head midline. The skull bone overlying the parietal cortex was removed with a high-speed drill to form an ellipsoidal cranial window (8.0 mm-major axis and 6.0 mm-minor axis). The cranial window was bathed with normal saline. The end of reflectance fiber probe was placed on the exposed cortex with care taken to avoid large blood vessels. A burr hole ( $2 \mathrm{~mm}$ diameter) was drilled in the ipsilateral frontal bone as a site for contacting cortex with $3 \mathrm{M} \mathrm{KCl}$ solution. The center-tocenter distance between the burr hole and the reflectance fiber probes was $9.3 \mathrm{~mm}$. CSD was induced by applying a droplet of $\mathrm{KCl}$ solution to the burr hole. Extracellular local field potential (LFP) was recorded with a single $\mathrm{Ag} / \mathrm{AgCl}$ electrode with ball-shaped tip (tip diameter is $1 \mathrm{~mm}$ ). The recording electrode was placed on the cortex at the anterior to the edge of reflectance fiber probe with care taken to avoid large blood vessels. The center-tocenter distance between the burr hole and the recording electrode was $6.9 \mathrm{~mm}$. $\mathrm{Ag} / \mathrm{AgCl}$ reference electrode (RC5, World Precision Instruments) was placed in the neck muscle. Fig. $16 \mathrm{~b}$ shows the schematic illustration of experimental setup. Measurements of $R_{c}(\lambda)$ and $R_{s}(\lambda)$ were obtained simultaneously in the wavelength range from 500 to $900 \mathrm{~nm}$ at $10 \mathrm{sec}$ intervals for $60 \mathrm{~min}$. The signal of LFP was amplified at $1-100 \mathrm{~Hz}$ with a differential amplifier (DAM50, World Precision Instruments) and digitized at $5 \mathrm{~Hz}$ using the oscilloscope (TDS1000C-EDU, Tektronix) connected to a personal computer running the Open Choice software. 


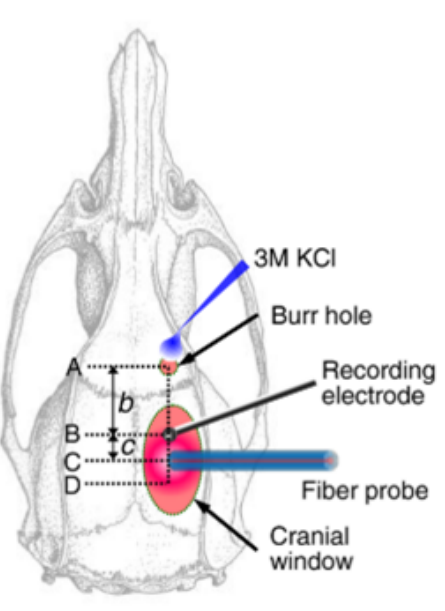

(a)
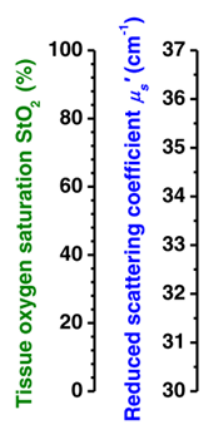
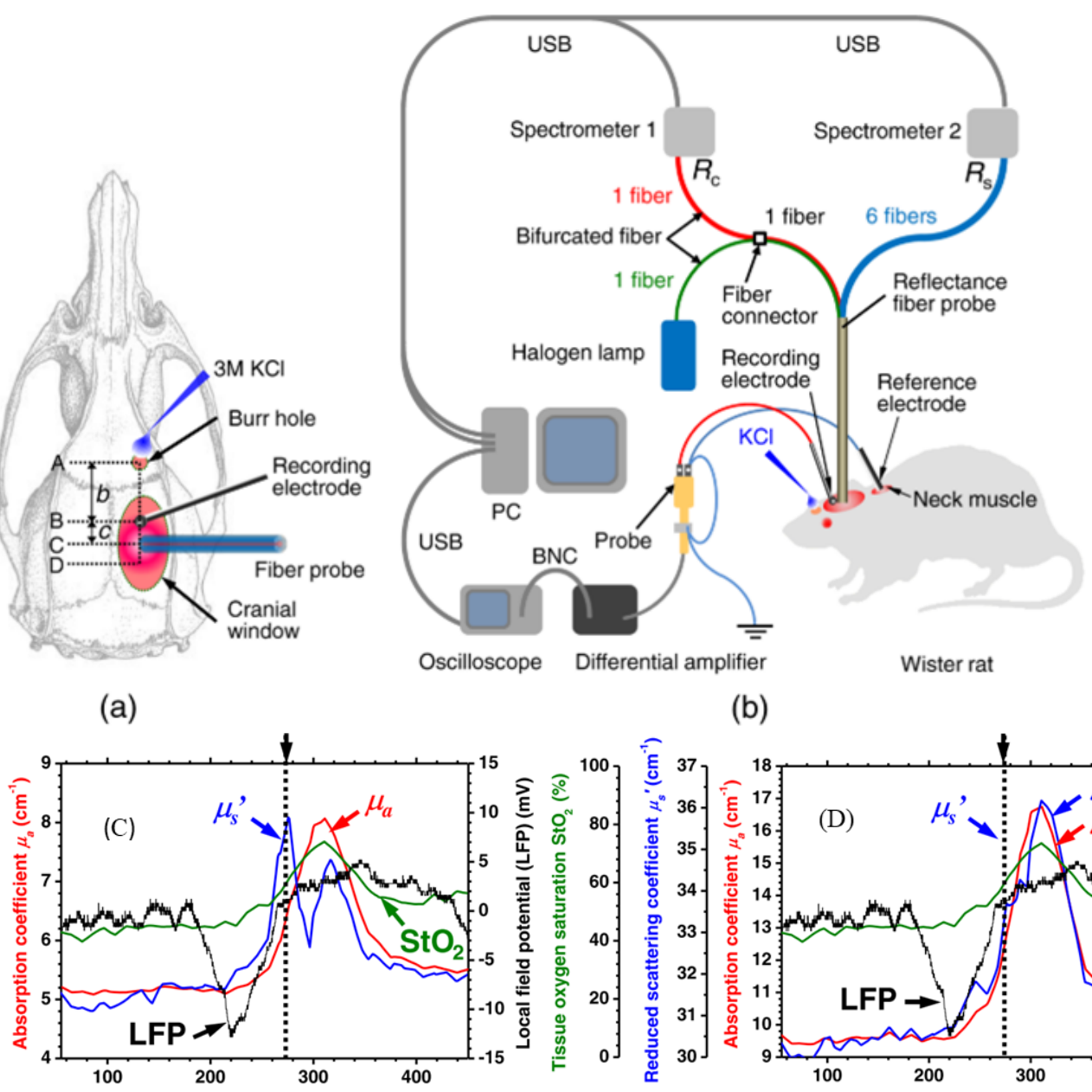

(b)
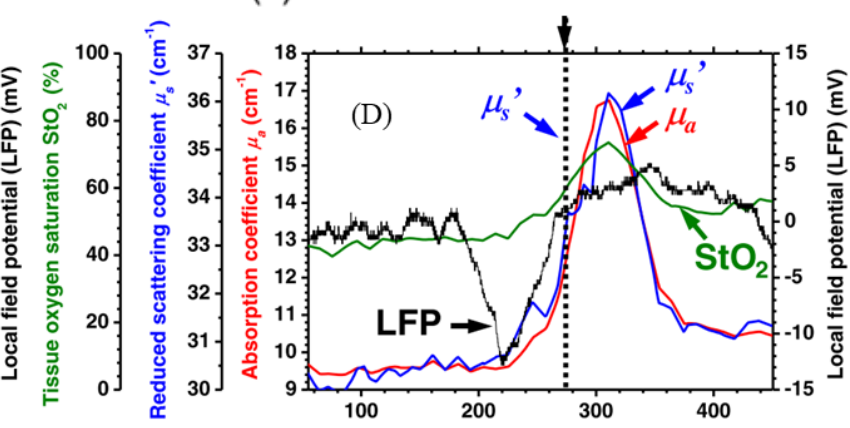

Fig. 16 Schematic illustration of stimulating (A) and measuring (B-C-D) sites on the rat head (a), experimental setup (b) for in vivo rat brain studies during cortical spreading depression, and experimental results for time course of reduced scattering coefficient $\mu_{s}^{\prime}$ and absorption coefficient $\mu_{a}$ at $500 \mathrm{~nm}$ (c) and $570 \mathrm{~nm}(\mathrm{~d})$, tissue oxygen saturation $\mathrm{StO}_{2}$ and local field potential (LFP) after the application of KCl. Reproduced from Ref. [41]. Published by SPIE.

Figs $16 \mathrm{c}$ and $16 \mathrm{~d}$ show the time courses of $\mu_{s}^{\prime}$ and $\mu_{a}$ for the specific wavelengths (500 $\mathrm{nm}$ (c) and $570 \mathrm{~nm}(\mathrm{~d})$ ), $\mathrm{StO}_{2}$ and LFP during CSD. The negative peak of LFP was observed at $158 \mathrm{sec}$ after the application of $\mathrm{KCl}$. Assuming that the time from the application of $\mathrm{KCl}$ to the negative peak of LFP is the propagation time of CSD, the propagation speed of the first CSD was $2.62 \mathrm{~mm} / \mathrm{min}$. The arrival time of CSD at the position under the reflectance fiber probe was expected to be $t=272 \mathrm{sec}$ (dashed lines in Figs. 16c and 16d) from the propagation speed of CSD and the distance between the reflectance fiber probe and the recording electrode. Temporal changes in $\mu_{a}$ in both Figs. 16c and 16d are indicative of the hemodynamic response to CSD. The value of $\mu_{s}^{\prime}$ at $570 \mathrm{~nm}$ during CSD is well correlated with that of $\mu_{a}$ (Fig. 16d). In the wavelength range of 500-570 nm, light is strongly absorbed by hemoglobin and scattered by red blood cells. Therefore, the changes in $\mu_{s}^{\prime}$ at $570 \mathrm{~nm}$ may reflect the scattering by red blood cells due to hemodynamics change in cerebral cortex. On the other hand, $\mu_{s}^{\prime}$ at $500 \mathrm{~nm}$ (Fig. 16c) peaked at $t=276 \mathrm{sec}$ before the profound increase in $\mu_{a}$ and corresponded to the expected arrival time of CSD at the position under the reflectance fiber probe. The DC shift of LFP is coincident with a rise in extracellular potassium and can evoke cell deformation generated by water movement between intracellular and extracellular compartments, and hence the light scattering by tissue. Therefore, the increase in $\mu_{s}^{\prime}$ at $500 \mathrm{~nm}$ before the profound increase in $\mu_{a}$ is indicative of changes in light scattering by tissue. This indicates a potential of the method to evaluate the depolarization of in vivo brain. The tissue oxygen saturation $\mathrm{StO}_{2}$ suddenly decreased followed a profound increase. This response of $\mathrm{StO}_{2}$ to application of $\mathrm{KCl}$ may indicate the temporal change of arterial blood flow due to CSD [42].

In the study [43], a method for estimation of the absorption coefficient in brain tissue based on diffuse reflectance spectra has been developed. Hemoglobin content can give information about blood volume in the tissue. Caution should be taken regarding this when using an invasive probe, because it is likely to affect the blood distribution in the tissue when inserted. The pressure from the insertion could press out blood from the vessels in the tissue, and bleeding could cause the presence of blood between the probe and the tissue. The substantia 
nigra lies just beneath the subthalamic nucleus (STN), which is a common target for deep brain stimulation (DBS), and the presence of melanin in a measured spectrum could thus indicate a too deep position for a DBS electrode that is supposed to be in the STN. In the cortex spectrum, the concentrations for cytochromes $\mathrm{c}$ and aa3 were estimated to be 2.2 and $4.0 \mathrm{M}$, respectively. In comparison, in in vitro measurements for human frontal cortex of Parkinsonian brains cytochrome volume concentrations were estimated as of $2.6 \mathrm{M}$ for cytochrome $\mathrm{c}$ and $2.0 \mathrm{M}$ for cytochrome aa3. The proposed method will give an approximate quantitative measurement of the absorption coefficient and should thus allow for quantification of chromophores in the brain. This study also elucidates the importance of taking the impact of the absorption coefficient into account when estimating the apparent photon path lengths [43].

\section{Modern methods of spreading light in tissues}

\subsection{Used Visible Chinese Human brain model}

The Visible Chinese Human (VCH) head model contains various types of tissues in a Chinese male human whole body frozen in the standing posture, and was horizontally sectioned at $0.2 \mathrm{~mm}$ interval [7]. The $0.1 \mathrm{~mm} /$ pixel resolution photographs were obtained with digital color, which is higher than $\mathrm{CT}$ and MRI resolution. The sections of VCH are performed by color photographs (see Fig. 17a). Distinct tissues in each slice were visibly distinguished, segmented and labeled with a series of specified number (see Fig. 17b). In the analysis of the color photographs and the numbered sections, the head model was segmented into eight types of tissue, including scalp, skull, CSF, muscle, visible artery, vein blood vessels, gray and white matter. The optical features of these tissues at 660, 810, and $980 \mathrm{~nm}$ are listed in (Table 2).

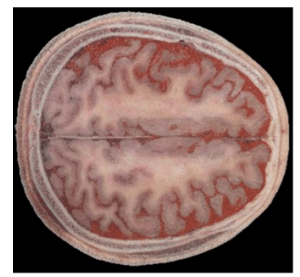

(a)

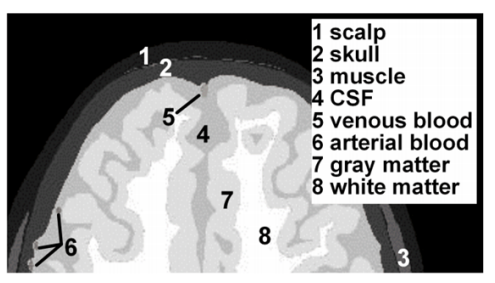

(b)
Fig. $17 \mathrm{VCH}$ head model: digital color photograph of one typical slice in the VCH head dataset (a); segmentation of the slice in (a) and identification of different types of tissues (b). Reproduced from Ref. [7]. Copyright 2017, The Authors. Published by World Scientific.

Using a statistical analysis of the characteristic parameters affecting the 3D light distribution, an estimate of the light penetration depth was obtained for various types of beam, sizes and wavelengths. The position in the center of the forehead, which is $1 \mathrm{~cm}$ above the eyebrow, is ideal for placing the center of the beam when it falls normally.

The optical properties of gray and white matter prove that visible and NIR light penetrates deep into brain tissue. It is obvious that wavelength plays a more important role in determining light penetration depth than beam type. It is found that $810 \mathrm{~nm}$ is more suitable than $660 \mathrm{~nm}$ and $980 \mathrm{~nm}$ in transcranial LLLT for more deeply and widely penetration. Besides, it is found that for $810 \mathrm{~nm}$, light could reach more widespread region in human brain compared to the other two wavelengths (see Fig. 18) [7].

Table 2 Optical properties of head tissues for the wavelengths 660,810 and $980 \mathrm{~nm}$. The units of both absorption coefficient $\left(\mu_{a}\right)$ and scattering coefficient $\left(\mu_{s}\right)$ are $\mathrm{cm}^{-1}$. For gray and white matter $\mu_{s}^{\prime}=\mu_{s}(1-g)$ is shown [7].

\begin{tabular}{cccc} 
Wavelength (nm) & $\mathbf{6 6 0} \boldsymbol{\mu}_{a} / \boldsymbol{\mu}_{s}$ & $\mathbf{8 1 0} \boldsymbol{\mu}_{a} / \boldsymbol{\mu}_{s}$ & $\mathbf{9 8 0} \boldsymbol{\mu}_{a} / \boldsymbol{\mu}_{s}$ \\
\hline Scalp & $0.340 / 25.8$ & $0.195 / 19.2$ & $0.450 / 14.0$ \\
\hline CSF & $0.040 / 3.5$ & $0.050 / 2.4$ & $4.5 / 1$ \\
\hline Skull & $0.300 / 24.3$ & $0.165 / 15.6$ & $0.360 / 11.5$ \\
\hline Muscle & $0.868 / 8.61$ & $0.273 / 6.87$ & $0.547 / 5.65$ \\
\hline Gray matter & $0.22 /(88(1-0.85))$ & $0.3 /(70(1-0.892))$ & $0.5 /(55(1-0.9))$ \\
\hline White matter & $0.82 /(400(1-0.835))$ & $1 /(350(1-0.862))$ & $1.4 /(300(1-0.88))$ \\
\hline Arterial blood & $1.30 / 61.1$ & $2.33 / 50$ & $2.90 / 37.4$ \\
\hline Venous blood & $4.87 / 25.0$ & $2.38 / 52.2$ & $1.62 / 5.15$
\end{tabular}



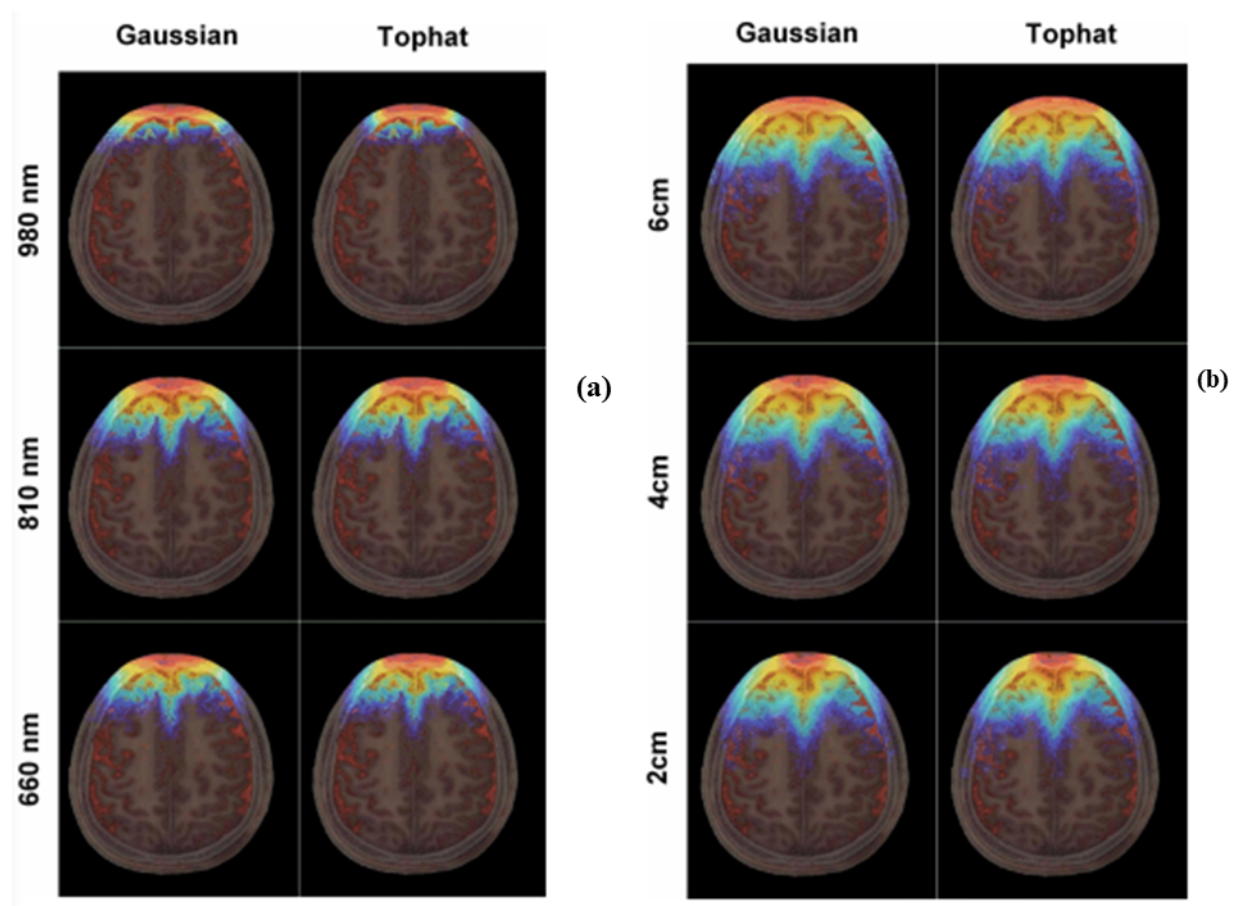

Fig. 18 Light fluence distribution with the background of head geometry and 3D structure shown for Gaussian and Tophat light beams: the LLLT fluence distribution respective to head geometry at different wavelengths $(660,810$, and $980 \mathrm{~nm})$ and for $4 \mathrm{~cm}$ light beam diameter (a); the LLLT fluence distribution at $810 \mathrm{~nm}$ for different light beam diameters $(2,4$, and $6 \mathrm{~cm}$ ) (b). Reproduced from Ref. [7]. Copyright 2017, The Authors. Published by World Scientific.

The pie chart (Fig. 19) suggests the percentages of fluence in each tissue that detected in head tissue layers, such as scalp (skin + muscle), skull, CSF, brain (gray and white), and blood. Moreover, the percentage of fluence in gray and white matter showed that light penetrates deep into the brain tissue.

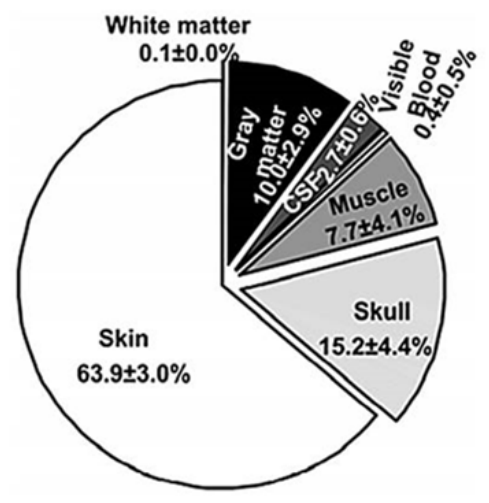

Fig. 19 Percentage of light fluence distribution in each type of tissue in $\mathrm{VCH}$ head model under normal condition. Data are shown in average statistics among different emitting positions on the forehead. Reproduced from Ref. [7]. Copyright 2017, The Authors. Published by World Scientific.

\subsection{Effect of scalp hair follicles on NIRS quantification}

Near-infrared spectroscopy (NIRS) has increasingly been used to noninvasively determine hemodynamic concentration change noninvasively by detecting light intensity changes [22-24, 44]. The effect of scalp hair follicle (SHF) on NIRS quantification is highlighted since its dark pigmentations is a strong absorption source to contaminate the NIRS signal. The Monte Carlo modeling for light transport in voxelized media (MCVM) and $\mathrm{VCH}$ head model with high precision in depicting 3D human anatomical structures were used to study the effect of SHF density on NIRS quantification (Fig. 20).
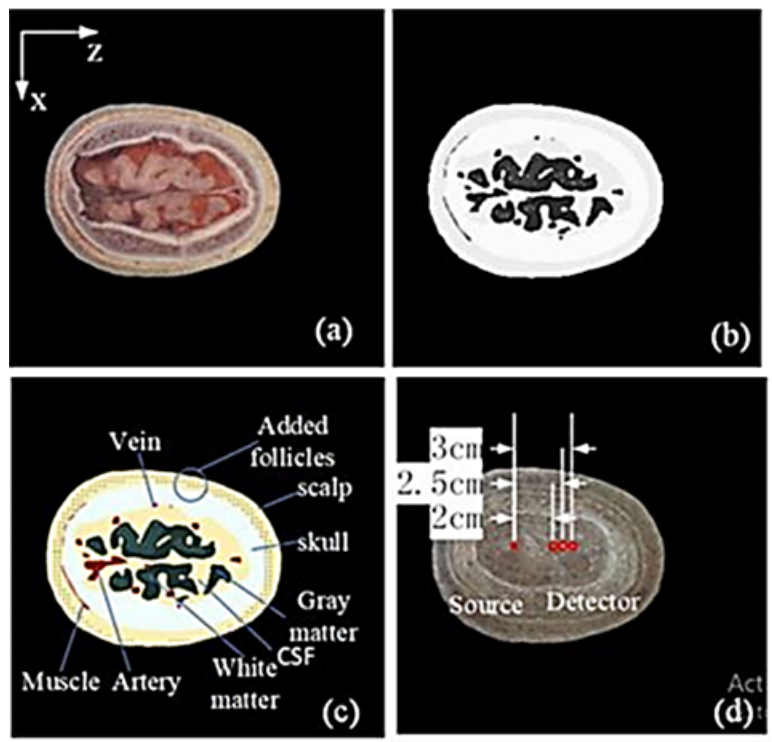

Fig. $20 \mathrm{VCH}$ model and model processing: original VCH model figure (a); after gray scaling process (b); after multi-thresholding distinction process (c); arrangement of source and detectors (d). Images adapted from Ref. [44]. 
The study [44] simulated the effect of scalp hair follicle with various densities on the detected intensity. Detected intensity changes when activation of a functional brain area occurs and hemodynamic parameters change correspondingly. The influence of SHF is significant. The finding of the linear correlation between NIRS measurement underestimation and the density of SHF also indicates a potential calibration method to eliminate the effect on NIRS measurement. The optical property of the follicle model only focused on the Asian population which means the modulation of follicle optical properties is based on black and gray hair shaft. Follicle with lighter colored hair may have less effect than reported [44].

\subsection{Source localization techniques in diffuse optical tomography for $f$ NIRS}

Functional near-infrared spectroscopy (fNIRS) is a noninvasive imaging technique that elicits growing interest for research and clinical applications [22-24, 44-47]. In the last decade, efforts have been made to develop a mathematical framework in order to image the effective sources of hemoglobin variations in brain tissues. Although the temporal resolution of fNIRS is higher than it is for fMRI, its spatial resolution is relatively low $(\sim 5$ to $10 \mathrm{~mm}$ ) with a depth of $\sim 2$ to $3 \mathrm{~cm}$ into the cortical surface due to the limited penetration of diffuse light sources into tissues. The fNIRS measurements can be used to infer images in $3 \mathrm{D}$, reflecting those areas where the light has undergone changes in intensity, a technique called diffuse optical tomography (DOT) [45].

Other authors [46] explain effect of human brain edema on light propagation, where brain edema originates from the excessive accumulation of CSF in the brain attributing to brain trauma or non-trauma diseases such as cancer, ischemic stroke, meningitis, and encephalitis. The high intracranial pressure could extrude the vessels in brain and block the blood circulation, yielding the risk of intracranial hemorrhage (ICH). In this study, the feasibility of utilizing fNIRS for brain edema inspection was investigated through Monte Carlo simulations over the head model of the $\mathrm{VCH}$ dataset. With the increase of CSF volume, the results show a strong linear relationship between the volumes of CSF and the intensities of the detected signal and the deeper penetration of photons. An outstanding contrast were also observed before and after the occurrence of ICH.

The NIRS offers a simple, fast, portable, relatively inexpensive method for noninvasive deep tissue hemodynamic monitoring. The probing depth of fNIRS can be as large as centimeters, sufficient for penetrating the skull and reaching the human brain. Moreover, the differentiations in optical properties and oxygenation between blood and CSF make possible to be aware of the presence of blood in CSF instantly.

Using MCVM simulations and phantom studies, it was shown that the change in the detected light was mainly due to the change in the thickness of the CSF, and not the gray and white matter. Performed simulations allowed for investigation of the alternations in different development levels of brain edema in such as absorption, fluence, and spatial sensitivity distribution (SSD) in head comprehensively instead of light intensity alone. Also, to inspect the light variation caused by $\mathrm{ICH}$. The results confirmed that the contrasts in optical signal can well reflect the changes in CSF volume and the presence of excessive blood in CSF.

Muscle took very minor proportion in head components so that SSD of muscle responded little with the edema level change. Increases were observed in CSF and white matter due to the volume increase of CSF and deep photon propagation. However, the absolute SSD proportion of white matter was too small $(<1 \%)$ to be a reliable criterion for brain edema assessment. The skull, blood, and scalp did not participate in the growth of CSF and their SSD proportion decreased owing to the increase of total SSD caused by deeper propagation as well. The growth of CSF shrank the proportion of gray matter in the detection path of fNIRS, yielding the SSD decline of gray matter. Brain edema may not only occur in CSF but also in gray matter and white matter. In practical utilization, fNIRS signal may be required to reach deeper layers. The penetration depth of fNIRS is roughly half of the source-detector (S-D) separation. In the study [46], the $\mathrm{S}-\mathrm{D}$ was set at $35 \mathrm{~mm}$ allowing for about $18 \mathrm{~mm}$ penetration. This depth made the photon sufficiently explore the CSF and a part of gray matter. This encouraging finding indicates that fNIRS also holds prospect to inspect the combination of brain edema in CSF as well as gray matter (see Table 3 ).

Table 3 Optical properties of tissue components at $800 \mathrm{~nm}$ [41].

\begin{tabular}{ccccc}
$\begin{array}{c}\text { Tissue } \\
\text { type }\end{array}$ & $\begin{array}{c}\boldsymbol{\mu}_{\boldsymbol{a}} \\
\left(\mathbf{c m}^{-\mathbf{1}}\right)\end{array}$ & $\begin{array}{c}\boldsymbol{\mu}_{\boldsymbol{s}} \\
\left(\mathbf{c m}^{-\mathbf{1}}\right)\end{array}$ & $\boldsymbol{g}$ & $\boldsymbol{n}$ \\
\hline Scalp & 0.10 & 187 & 0.81 & 1.37 \\
\hline Skull & 0.16 & 160 & 0.90 & 1.43 \\
\hline Muscle & 1.40 & 500 & 0.90 & 1.40 \\
\hline CSF & 0.04 & 24 & 0.90 & 1.33 \\
\hline $\begin{array}{c}\text { Gray } \\
\text { matter }\end{array}$ & 0.36 & 220 & 0.90 & 1.37 \\
\hline $\begin{array}{c}\text { White } \\
\text { matter }\end{array}$ & 0.14 & 910 & 0.90 & 1.37 \\
\hline $\begin{array}{c}\text { Blood } \\
\text { Mlo }\end{array}$ & 2.35 & 512 & 0.99 & 1.40
\end{tabular}

Light intensity was found to be straightforwardly correlated with the volume increase of CSF. Photons can reach the cerebral tissue. The occurrence of $\mathrm{ICH}$ can outstandingly produce lower intensity, which can be used as a reflection on the presence of blood in CSF. Overall, this study demonstrated the great potential of fNIRS in monitoring brain edema [46].

Continuous wave fNIRS non-invasively interrogates local changes in brain hemodynamics and thus can 
provide functional brain imaging [47]. Light interaction with chromophores and scatterers presents in the head tissues leaves a spectroscopic attenuation signature in the exiting radiation from where the concentration of the chromophores can be estimated; a mathematical effort known as image reconstruction. Scalp blood flow contamination is one of the most pressing issues affecting fNIRS data reconstruction. The scalp comes first in the optical path and it is visited twice during the photon migration. Reported a new forward model for the estimation of the diffuse reflectance in the adult human head [47]. Previous work has considered only 4 layers (scalp, skull, CSF, grey, and white matter) as 9 layers are considered in Ref. [47]. The new model emphasizes the detail consideration for the scalp sublayers, so that attenuation due to scalp blood flow can be characterized for latter incorporation to the reconstruction algorithm [47].

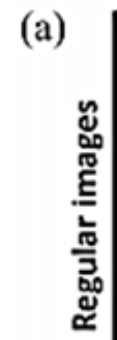

(c)
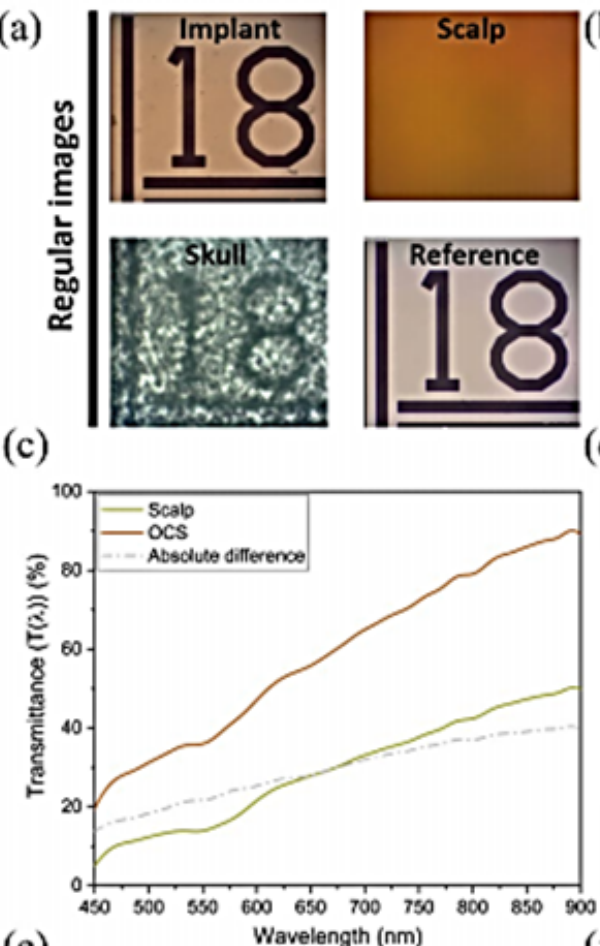

(e)

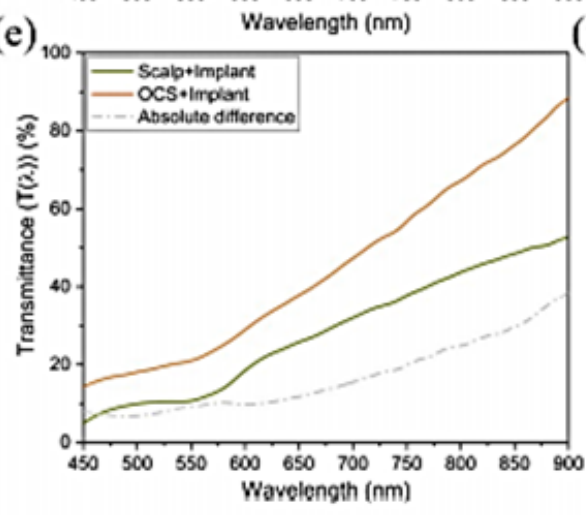

\subsection{Theranostic cranial implant for hyperspectral light delivery and microcirculation imaging}

From the deep blue to the NIR is the region among the wide spectrum of electromagnetic waves that know as light (Fig. 1). This delicate region has a distinct advantage for interrogating biological tissue due to its unique photon energy range of $0.5-3 \mathrm{eV}$. At energies above this range, $\mathrm{C}-\mathrm{C}$ and $\mathrm{C}-\mathrm{H}$ bonds dissociate and ionization can occur, while at energies below this range, water absorption dominates the transmittance, preventing any specific targeting of molecules. The noninvasive interactions with tissue of photons within this energy range have been used in numerous approaches to assess brain health and treat its diseases.

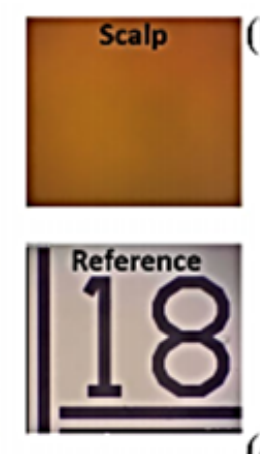

(d)
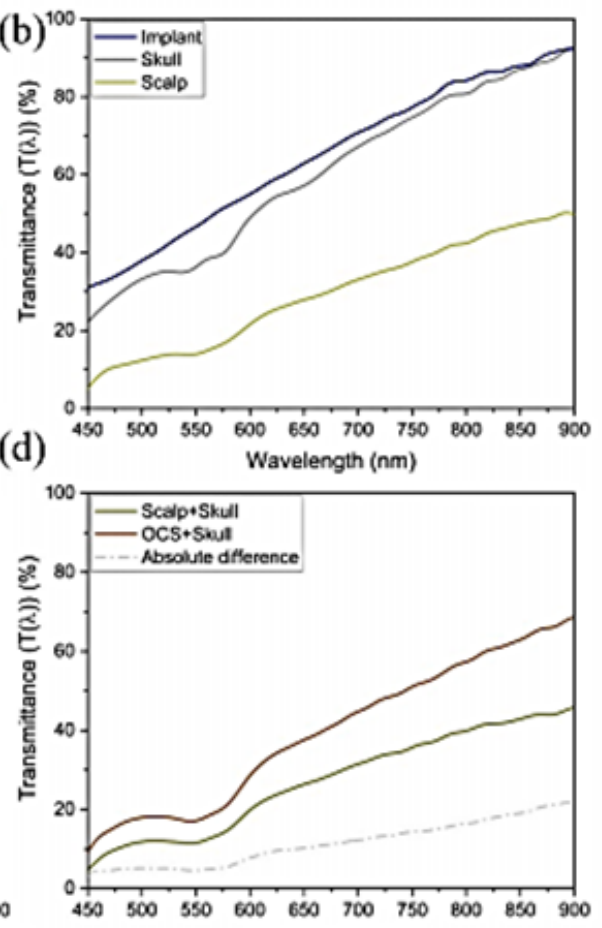

(f)

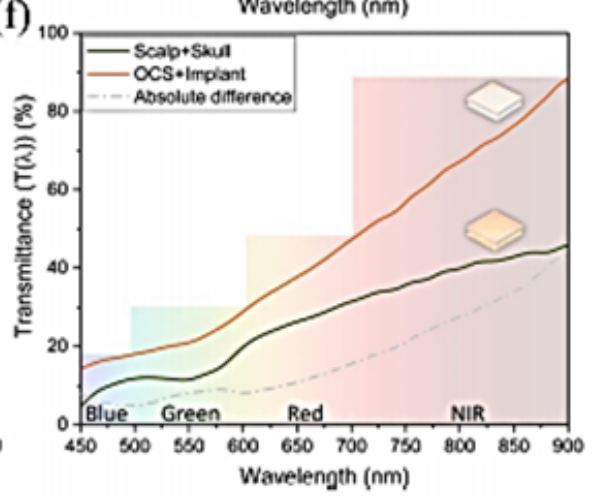

Fig. 21 Visual comparison of light transmission using photographs of an NBS 1963A resolution target through the WttB implant, skull, and scalp: the resolutions shown are the 18 cycle/mm target (each black line width is $27.78 \mu \mathrm{m})(\mathrm{a})$; optical transmittance of scalp, skull, and implant (b); comparison of optical transmittance through scalp and OCS (c); comparison of the effect of optical clearing on optical transmittance of the tissue sample stacks (d-f): Scalp + Skull vs. OCS + Skull (d), Scalp + Implant vs. OCS + Implant (e), Scalp + Skull vs. OCS + Implant (f). Reproduced from Ref. [48]. Copyright 2019, The Authors. Published by "bioRxiv". 
The application of transparent cranial implants holds the transformative potential for facilitating the diagnosis and treatment of a wide variety of brain pathologies and neurological disorders [48]. Implementing this implant in the current cranial prostheses or a separate implantation of "Window to the Brain" (WttB) implant (e.g. on a drilled hole in the skull) enables delivery to and/or acquisition of light from the brain, in real-time, without the need for scalp removal for various post-operation applications. In the study [48], the light delivery improvement by WttB implant coupled with optical cleared scalp (OCS) in a set of ex vivo optical characterization measurements was assessed. Additionally, the feasibility of through-scalp brain microcirculation imaging using the implant in vivo was evaluated. A mixture of two biocompatible chemicals, PEG-400 (PEG) and Propylene Glycol (PG) were used as scattering reducer and penetration enhancer, respectively, at a volume ratio of 9:1 at room temperature.

The pristine scalp tissue has a very low light transmission, the implant has a higher optical transmittance (up to $12 \%$ ) than skull. It should also be noted that while the optical transmittance of the implant is an improvement over the skull, the mouse skull is inherently transparent itself, which is not the case in larger animals or humans. The scalp showed a lower transmittance compared to skull and implant (maximum difference of $39 \%$ ). This indicates that the scalp is the limiting factor for light transmission within the tissues above the mouse brain. As the size of vessels and velocity of blood flow is not expected to differ on average between the imaging conditions, the apparent increase in signal-to-noise ratio (SNR) and vessel diameter imaged through the scalp, skull, implant, and OCS + implant is likely due to the blurring of the image (see Fig. 21).

The skull and OCS scattering both disorder the speckle pattern that was created by the brain hemodynamics. The reduction in border sharpness of the vessels imaged through the intact skull vs. OCS + implant suggests that the skull has a higher blurring effect [48].

Since the removal of dura invariably promotes tissue swelling, small craniotomies maintain tissue architecture better. Large craniotomies provide multiple imaging sites within the window and facilitate assessment of spatial patterns in functional processes of interest. However, as size of the window increases, tissue disruption also increases. Diameter window of $6 \mathrm{~mm}$ was found to be well suited for experimental requirements [49]. Tis size provided long term patency of the window, while still fitting over one cortical hemisphere. Although the thinned skull method has allowed in vivo TPFM to cortical depths from $250 \mu \mathrm{m}$ to $400 \mu \mathrm{m}$ in the mouse, the thickness of rat meninges precludes microscopy of blood flow dynamics or neuronal activity below the pial surface. Tissue swelling may be controlled by gentle pressure on the glass coverslip, which helps re-establish intracranial pressure post cranial window implantation (see Fig. 22).

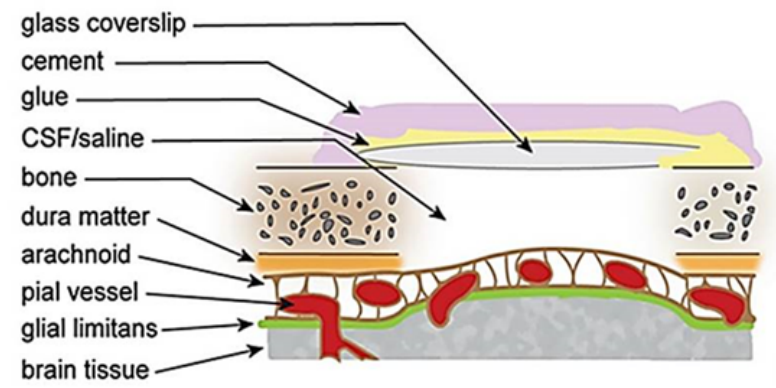

Fig. 22 Cross-sectional rendering of the craniotomy and tissue layers affected in the surgical procedure. The technique used for placement of the glass coverslip (skull surface, thinned skull, or adjacent to the pial surface) will modify application of cementing compound, the degree of distortion of pial surface, and the extent of inflammation below the coverslip. Coverslip placement on skull surface is indicated (not to scale). Images adapted from Ref. [49].

A 3D reconstruction of the vascular architecture at 10 weeks post-surgical implant allowed visualization of cortical penetrating arteries and veins, as well as arterioles, capillaries and venules. Excellent intra- vs. extra-vascular contrast was observed in Layer I, encompassing 80-100 $\mu \mathrm{m}$ below the pial surface, Layer II/III 200-580 $\mu \mathrm{m}$, and finally reaching the superficial region of Layer IV around $600 \mu \mathrm{m}$ below cortical surface. Using progressive increases in laser intensity with depth, the signal-to-background ratio (SBR) remained relatively constant until $400 \mu \mathrm{m}$ in cortical depth [49].

Voltage-sensitive dye (VSD) imaging is a powerful tool enabling visualization of the network activity of thousands of neurons at high spatiotemporal resolution. However, VSD imaging usually requires removal of the dura mater for dye staining, and thereafter the exposed cortex needs to be protected using an optically transparent artificial dura.

The objective of the study [50] was to apply a collagen membrane as an artificial dura substitute for VSD imaging. The membrane had superior characteristics in chemical and optical transparency and maintained the cortical surface in good condition after implantation. VSD signals report inhibitory as well as excitatory neural activity, with positive and negative values, respectively. The study evaluated the collagen dura membrane in experiments using anesthetized rats. Therefore, next step is to perform VSD imaging in freely behaving animals. As the implantable cranial window device was fabricated using a 3D printer, it will be easy to modify the outer shape to fit the fiberoptic periscope/microscope terminus.

After replacing the native dura with a transparent dura, several studies have already reported successful injection of a virus and transdural illumination through the artificial dura. Because used membrane is thinner (35 $\mu \mathrm{m}$ vs. $100-200 \mu \mathrm{m})$, the viral injection procedure with glass pipettes should be easier [50]. 
In another study [51], the enhancement in optical access to the brain is evaluated upon comparing ex vivo transmittance measurements of mice native skull and the a transparent Yttria-stabilized zirconia (YSZ) cranial implant with scalp and OCAs. An increase in transmittance of up to $50 \%$ and attenuation lengths of up to $2.4 \mathrm{~mm}$ (i.e., a five-fold increase in light penetration) was achieved. The use of this ceramic implant and the biocompatible OCAs offer attractive features for NIR optical techniques for brain theranostics. The transmittance measurements show that the YSZ implant is more transparent throughout the tested NIR range (900-2400 nm) including all of the optical windows commonly used for biophotonic applications (i.e., NIR I, NIR II, SWIR I and SWIR II). This is due to the significant reduction in scattering and absorption when using the YSZ implant. In terms of relative differences in the optical properties between skull and the implant, the latter improves the light transmission within the water absorption bands owing to its reduced water content compared to the native skull $(70-75 \%)$. The skull transmittance shows the typical water absorption bands in the NIR spectral region (1450 nm and $1940 \mathrm{~nm}$ ), while the YSZ implant shows improved transmittance in these bands.

Although the scalp stacking leads to a reduction in transmittance of at least $60 \%$, the sample with the implant still provides the best performance, as confirmed by the attenuation length. Throughout all the spectral range, the sample formed with the scalp and the YSZ implant provides improved light penetration. The effects of applying OCAs on the scalp are evident, the transmittance spectra increases by $20 \%$ for the sample with the skull, while for the YSZ implant the enhancement is $40 \%$, the attenuation length for both samples increases accordingly; when compared to the results, it is clear that the OCAs effectively enhance the light penetration yielding larger attenuation lengths throughout the whole NIR range for both samples [51].

Laser speckle imaging (LSI) of mouse cerebral blood flow was compared through a transparent YSZ cranial implant over time (at days 0,14 , and $28, \mathrm{n}=3$ mice) vs. LSI through native skull (at day $60, \mathrm{n}=1$ mouse). The average sharpness of imaged vessels was found to remain stable, with relative change in sharpness under $7.69 \% \pm 1.2 \%$ over 28 days. Through-implant images of vessels at day 60 appeared sharper and smaller on average, with microvessels clearly visible, compared to through-skull images where vessels appeared blurred and distorted. These results suggest that long-term imaging through this implant is feasible [52].

To establish effective far field communications with a cortical backscattering implant, there was recently proposed a spatially distributed split-ring resonator inspired antenna system composed of a small thin and flexible implantable part that couples to a head-worn part acting as a performance booster [53]. Human body is an extremely complex operation environment for antennas, and thus computational electromagnetics and appropriate human body models are indispensable in the assessment of the electromagnetic performance of implantable and wearable antennas. These experiments ensure the feasibility of antenna system as a wireless platform in brain care applications further. Ongoing and future work focuses on integrating physiological sensors into the evaluated antenna system and development of a wearable part that provides circular polarization [53].

\section{Phototherapy for brain diseases}

\subsection{Optical monitoring of electric deep brain stimulation (DBS)}

The authors [54] have done quantification of blood fraction ( $\left.f_{\text {blood}}\right)$, blood oxygenation $\left(\mathrm{SO}_{2}\right)$, melanin, lipofuscin and oxidized and reduced cytochrome aa3 and c was done from diffuse reflectance spectra captured in cortex, white matter, globus pallidus internus (GPi) and subthalamus during stereotactic implantations of 29 deep brain stimulation (DBS) electrodes with the aim of investigating whether the chromophores can give physiological information about the targets for DBS. Double-sided Mann-Whitney U-tests showed more lipofuscin in GPi compared to white matter and subthalamus $(\mathrm{p}<0.05)$. Compared to the other structures,

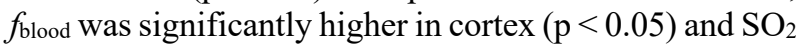
lower in GPi $(\mathrm{p}<0.05)$. The measurements indicate very low oxygenation and blood volume for DBS patients, especially in the GPi. In DBS, implanted electrodes are used to disrupt the pathologic neural activity in certain deep brain structures such as the subthalamic nucleus (STN) and zona incerta (ZI) in the subthalamus for Parkinson's disease (PD), the ventral intermediate nucleus in the thalamus for essential tremor or the GPi for dystonia.

In the study [54], diffuse reflectance spectroscopy (DRS) was used for examination of chromophore content in brain tissue. A method previously developed for estimation of these chromophores in the brain [43] was applied for reflectance spectra captured on patients undergoing DBS implantation. The aim of this study was to estimate and quantify chromophores from DRS captured in the cortex, white matter and target areas during intracerebral recordings in relation to implantation of DBS electrodes. Chromophores in the cortex, white matter, GPi and STN/ZI of patients undergoing DBS surgery for movement disorders were quantified from DRS. Very low estimated blood volumes and oxygen saturations were found in all structures, especially the GPi. This would indicate a hypoxic environment in the central brain of patients with PD or dystonia, though it is possible that the invasive nature of the measurements causes artificially low estimates [54].

\subsection{Nonpharmacological therapy of Alzheimer's disease}

The transcranial photobiomodulation (tPBM) is considered as a possible novel nonpharmacological and non-invasive therapeutic strategy for prevention or delay 
of $\mathrm{AD}$ [55]. In the last years, there is accumulating evidence suggesting that $\mathrm{PBM}$ can reduce amyloid $\beta$ $(\mathrm{A} \beta)$ peptide associated hippocampal neurodegeneration, memory impairments in rodents, inhibits $A \beta$-induced brain cell apoptosis and causes a reduction in $A \beta$ plaques in the cerebral cortex. Considering these facts, hypothesize that $\mathrm{PBM}$ due to a good penetration into the brain cortex and even into deep brain regions can stimulate meningeal lymphatic vessel dysfunction that might be one of the mechanisms underlings a discovered therapy of AD by $\mathrm{APBM}$.

Clinical data suggest that tPBM decreases $A \beta$ aggregates in human neoblastoma cells. In the peripheral tissues $\mathrm{TPBM}$ causes a reduction in the volume of tissues and anti-inflammatory effects probably due to nitric oxide (NO) modulation of lymphatic vessels constriction and lymph flow. There is the hypothesis that mitochondria might be initial events, which after light effects can increase synthesis of ATP, reactive oxygen species, intracellular $\mathrm{Ca}^{2+}$, and release NO. These data clearly show that $\mathrm{PBM}$ might be a promising novel therapeutic target for preventing or delaying AD [55].

\subsection{Optimizing a rodent model of Parkinson's disease for exploring the effects and mechanisms of deep brain stimulation}

DBS has become a treatment for a growing number of neurological and psychiatric disorders, especially for therapy-refractory PD [56]. However, not all of the symptoms of PD are sufficiently improved in all patients, and side effects may occur. Further progress depends on a deeper insight into the mechanisms of action of DBS in the context of disturbed brain circuits. For this, optimized animal models have to be developed. The numerical analysis of electric field effects aims at describing the distribution of the stimulated neurons around the DBS electrode based on the inhomogeneous current density and field distributions in the stimulated brain tissue. The induced transmembrane potential and, alternatively, the so-called "activation function", are considered the major determinants for neuronal stimulation.

Numerical calculations of electric potentials, electric fields, and current densities around DBS electrodes can be performed when dimensions and electric properties of the tissues that surround the electrodes are taken into account. Despite new and promising developments in the field of transgenic animal technology, the conventional 6-OHDA hemi-PD rat model is still suitable for the investigation of various aspects of experimental DBS, such as the analysis of electrochemical processes at the electrode/tissue interface and of molecular and cellular changes in the tissue surrounding the stimulating electrode. For optimization, new electrode materials and modified surface structures are investigated in combination with computational simulation and numerical electric field calculations. Also, new target regions are tested for effects of DBS on motor and cognitive functions assessed by specific behavioral tests.
The final aim is the improvement of the efficacy and safety of DBS in clinical practice [56].

\section{The light beams interaction with brain tissues}

\subsection{Vortex photons and transmission of classically entangled beams through mouse brain tissue}

Majorana-like photons arise from a class of vector photon beams, which are entangled states where the state and complex transpose are identical [57]. Majorana photons have within itself locally both right and left-handed chirality. The light transmission of Majorana and nonMajorana photon vortex beams were investigated in a chiral media with orbital angular momentum (OAM) in a mouse brain at different local regions showing enhanced transmission and properties of being entangled [57, 58]. As a result, a higher transmission change and moderate variation with respect to OAM values were observed with Majorana vector beams, which is attributed to their nonseparability property and dual chirality nature. Also, left circular polarization showed a largest variation as the OAM values increase due to the fact of being a pure circular polarization state.

Light transmission of Laguerre Gaussian (LG) vector vortex beams in different local regions in mouse brain tissue was investigated [58]. Transmittance was measured in the ballistic and diffusive regions with various polarization states and OAM. The transmission change observed with structured light other than linear polarization is attributed to chiroptical phenomena from the chiral brain media and the handedness of the light.

In optical imaging, light's salient properties such as polarization, wavelength, and spatial modes play an important role for imaging in scattering media. Scattering blurs images and the degree of scattering depends on wavelength. The polarization becomes randomized due to scattering. The study [58] focused on the transmission of $\mathrm{LG}$ vector vortex beams through mouse brain tissue in the ballistic $\left(z<l_{t r}=1 / \mu_{s}^{\prime}\right)$ and diffusive $\left(z>l_{t r}\right)$ regions, where $z$ is the thickness of the turbid medium and $l_{t r}$ is the transport mean free path of a photon. Different types of polarization at different special locations of the mouse brain at various thicknesses are investigated to demonstrate the role of classical entanglement in potentially improving imaging with higher flux of photons in the classical limit.

Vector beam study produced the salient outcome that the transmittance of LG beams through brain tissue displays strong positive dependency on OAM for collimated, radially, azimuthally and left circularly polarized LG beams. The classical entangled beam of radial and azimuthal polarization gave highest transmission as the OAM values increase in both ballistic and diffusive regions. Also, these vector beams gave clear trends when targeting different areas of the brain at the ballistic and diffusive region. Furthermore, radial polarization gave the highest transmission range at the 
diffusive region, while left circular polarization showed the highest transmission for the ballistic region. Moreover, in targeting the hippocampus, cortex, and white matter, radially polarized light proved to be the most effective followed by azimuthal polarized beams. On the other hand, linearly polarized light showed positive dependence on OAM, but this dependence proved to be less significant in comparison to the dependences on the other tested polarizations [58].

\subsection{Multi-wavelength time-resolved NIRS of the adult head}

Beside oxygenation studies, optical methods based on NIRS have been tested in combination with the application of an intravascular flow tracer in order to assess cerebral perfusion parameters. In particular, an optical technique based on diffuse reflectance measurement combined with indocyanine green (ICG) bolus tracking is extensively tested as a method for the clinical assessment of brain perfusion at the bedside [59, 60]. ICG is an optical contras agent of low toxicity revealing high absorption at the near-infrared wavelengths. After intravenous injection, ICG can be successfully monitored during its circulation through the brain. Similarly to the NIRS-based oxygenation studies, the technique based on monitoring of ICG bolus passage suffers from extracerebral contamination. In the study [59], broadband (spectral) time-resolved measurements in which the distributions of times of flight of photons DTOFs were recorded at 16 wavelengths simultaneously but at single source-detector separation $(\mathrm{S}-\mathrm{D}=3 \mathrm{~cm})$ were conducted during in vivo tests carried out in healthy volunteers. In phantom measurements, $\mathrm{S}-\mathrm{D}=1,2,3$, $4 \mathrm{~cm}$ during administration of a bolus of ICG. Presented results show that the changes in wavelength-resolved reflectance signals related to the inflow of ICG can be successfully detected. The number of photons, mean time of flight and variance of the DTOFs decreased with the increase of the tissue absorption related to the inflow of the dye [59].

\section{Conclusion}

Light penetration in the brain is subject to attenuation by several tissues (scalp, skull, dura mater, blood, and CSF).
Being able to quantitatively evaluate the light's energy attenuation by these tissues would lead to optimized tPBM parameters and could improve the outcome of brain tPBM therapy. Over the past two decades, researchers have simulated computer models and experimentally measured ex vivo animal and human tissues to try and define the best irradiation parameters for brain $\mathrm{TPBM}$. Previous studies have revealed that cortical light fluences within the range of $1-20 \mathrm{~J} / \mathrm{cm}^{2}$ are optimal to activate the neurobiological processes involved in neuroprotection and neural repair [61]. According to a recent publication, $810 \mathrm{~nm}$ was the most popular wavelength with $55.4 \%$ of reports, next was $660 \mathrm{~nm}$ with $14.4 \%, 1064 \mathrm{~nm}$ with $14.0 \%, 630 \mathrm{~nm}$ with $9.3 \%$, and finally $980 \mathrm{~nm}$ with $6.9 \%$ [18]. Of the reviewed wavelengths, $810 \mathrm{~nm}$ not only achieves the deepest penetration through the head tissues but also has the highest absorption by cytochrome c oxidase (CCO) making it best suited to deep brain tissues.

Studies in human cadaver models and mesh computerized head modeling have demonstrated that light penetrates up to $3-4 \mathrm{~cm}$ into the brain at very low level $[21,22,61]$. According to the prevailing theory of transcranial hinging on photochemical reactions, very low-level light is insufficient to produce biological effects; the brain organoid might provide a means to test if mechanistically-different biological effects could occur deeper in the brain as a result of transcranial light exposure. The reviewed literature suggests that larger thickness of intervening tissues between skin surface and brain and tissue dehydration-both typically associated with aging-is inversely correlated with light penetration. It is then anticipated that data on dosimetry and target engagement of brain function in young healthy subjects might underestimate effective dosimetry of TPBM for the middle aged and the elderly [61].

\section{Disclosures}

All authors declare that there is no conflict of interests in this paper.

\section{Acknowledgements}

VVT was supported by the Government of the Russian Federation via Grant No. 075-15-2019-1885.

\section{References}

1. S. Stubinger, F. Klampfl, M. Schmidt, and H. F. Zeilhofer, "Lasers in Oral and Maxillofacial Surgery," Springer Nature, Switzerland AG (2020).

2. S. Bordin-Aykroyd, R. B. Dias, and E. Lynch, "Laser-Tissue Interaction," EC Dental Science 18(9), 2303-2308 (2019).

3. O. Hamdy, H. S. Mohammed, "Investigating the transmission profiles of $808 \mathrm{~nm}$ laser through different regions of the rat's head," Lasers in Medical Science (2020).

4. S. Golovynskyi, I. Golovynska, L. I. Stepanova, O. I. Datsenko, L. Liu, J. Qu, and T. Y. Ohulchansky, "Optical windows for head tissues in near-infrared and short-wave infrared regions: Approaching transcranial light applications," Journal of Biophotonics 11(12), e201800141 (2018). 
5. A. N. Bashkatov, E. A. Genina, V. I. Kochubey, and V. V. Tuchin, "Optical properties of human skin, subcutaneous and mucous tissues in the wavelength range from 400 to 2000 nm," Journal of Physics D: Applied Physics 38(15), 2543-2555 (2005).

6. E. A. Genina, A. N. Bashkatov, D. K. Tuchina, A. Dyachenko, N. Nafulukin, A. Shirokov, A.Khorovodov, A. Terskov, M. Klimova, A. Mamedova, I. Blokhina, I. Agranovich, E. Zenchenko, O. V. Semiachkina-Glushkovskaya, and V. V. Tuchin, "Optical properties of brain tissues at the different stages of glioma development in rats: pilot study,” Biomedical Optics Express 10(10), 5182-5197 (2019).

7. T. Li, C. Xue, P. Wang, Y. Li, and L. Wu, "Photon penetration depth in human brain for light stimulation and treatment: A realistic Monte Carlo simulation study," Journal of Innovative Optical Health Sciences 10(5), 1743002 (2017).

8. V. V. Tuchin, "Tissue optics and photonics: Biological tissue structures," Journal of Biomedical Photonics \& Engineering 1(1), 3-21 (2015).

9. K. Kumar, K. Boone, J. Tuszynski, P. Barclay, and C. Simon, "Possible existence of optical communication channels in the brain," Scientific Reports (6), 36508 (2016).

10. P. Adams, F. Petruccione, “Quantum effects in the brain: A review,” AVS Quantum Science 2(16), 022901 (2020).

11. S. Wirdatmadja, P. Johari, A. Desai, Y. Bae, E. Stachowiak, M. Stachowiak, J. M. Jornet, and S. Balasubramaniam, "Analysis of light propagation on physiological properties of neurons for nanoscale optogenetics," IEEE Transactions on Neural Systems and Rehabilitation Engineering 10, 1534-4320 (2019).

12. K. Ning, X. Zhang, X. Gao, T. Jiang, H. Wang, S. Chen, A. Li, and J. Yuan, "Deep-learning-based whole-brain imaging at single-neuron resolution," Biomedical Optics Express 11(7), 3567-3584 (2020).

13. V. V. Tuchin, "Tissue optics and photonics: Light-tissue interaction," Journal of Biomedical Photonics \& Engineering 1(2), 98-134 (2015).

14. V. V. Tuchin, "Tissue optics and photonics: Light-tissue interaction II," Journal of Biomedical Photonics \& Engineering 2(3), 030201 (2016).

15. M. Gerstenmayer, B. Fellah, R. Magnin, E. Selingue, and B. Larrat, "Acoustic Transmission Factor through the Rat Skull as a Function of Body Mass, Frequency and Position," Ultrasound in Medicine \& Biology 44(11), 2336-2344 (2018).

16. G. Hong, S. Diao, J. Chang, A. L. Antaris, C. Chen, B. Zhang, S. Zhao, D. N. Atochin, P. L. Huang, K. I. Andreasson, C. G. Kuo, and H. Dai, "Through-skull fluorescence imaging of the brain in a new near-infrared window," Nature Photonics 8(9), 723-730 (2014) (accessed: December 2020 through https://europepmc.org).

17. A. Yu. Sdobnov, M. E. Darvin, E. A. Genina, A. N. Bashkatov, J. Lademann, and V. V. Tuchin, "Recent progress in tissue optical clearing for spectroscopic application," Spectrochimica Acta Part A: Molecular and Biomolecular Spectroscopy 197, 216-229 (2018).

18. P. Wang, T. Li, "Which wavelength is optimal for transcranial low-level laser stimulation?” Journal of Biophotonics 12(7), e201800173 (2019).

19. M. V. P. Sousa, R. Prates, I. T. Kato, C. P. Sabino, L. C. Suzuki, M. S. Ribeiro, and E. M. Yoshimura, "Laser scattering by Transcranial rat brain illumination," Proceeding of SPIE 8427, 842728 (2012).

20. P. A. Lapchak, P. D. Boitano, P. V. Butte, D. J. Fisher, T. Holscher, E. J. Ley, M. Nuno, A. H. Voie, and P. S. Rajput, "Transcranial Near-Infrared Laser Transmission (NILT) Profiles (800 nm): Systematic Comparison in Four Common Research Species," PLoS ONE 10(6), e0127580 (2015).

21. V. V. Lychagov, V. V. Tuchin, M. A. Vilensky, B. N. Reznik, Th. Ichim, and L. De. Taboada, "Experimental study of NIR transmittance of the human skull," Proceeding of SPIE 6085, 60850T (2006).

22. T. Myllylä, V. Yu. Toronov, J. Claassen, V. Kiviniemi, and V. V. Tuchin, "Near-infrared spectroscopy in multimodal brain research," Chapter 10 in Handbook of Optical Biomedical Diagnostics. Light-Tissue Interaction, V. V. Tuchin (ed.), SPIE Press, Bellingham, WA, USA, 687-735 (2016).

23. F. Tian, S. N. Hase, F. Gonzalez-Lima, and H. Liu, "Transcranial laser stimulation improves human cerebral oxygenation," Lasers in Surgery and Medicine 48(4), 343-349 (2016).

24. D. W. Barrett, F. Gonzalez-Lima, "Transcranial infrared laser stimulation produces beneficial cognitive and emotional effects in humans," Neuroscience 230, 13-23 (2013).

25. V. V. Tuchin, Optical Clearing of Tissues and Blood, SPIE Press, Bellingham, WA (2005).

26. E. A. Genina, A. N. Bashkatov, Yu. P. Sinichkin, I. Yu. Yanina, and V. V. Tuchin, "Optical clearing of biological tissues: prospects of application in medical diagnostics and phototherapy," Journal of Biomedical Photonics \& Engineering 1(1), 22-58 (2015).

27. L. Oliveira, V. V. Tuchin, The Optical Clearing Method: A New Tool for Clinical Practice and Biomedical Engineering, Springer Nature Switzerland AG (2019).

28. I. Costantini, R. Cicchi, L. Silvestri, F. Vanzi, and F. S. Pavone, "In-vivo and ex-vivo optical clearing methods for biological tissues: review," Biomedical Optics Express 10(10), 5251-5267 (2019).

29. E. A. Genina, Y. I. Surkov, I. A. Serebryakova, A. N. Bashkatov, V. V. Tuchin, and V. P. Zharov, "Rapid ultrasound optical clearing of human light and dark skin,” IEEE Transactions on Medical Imaging 39(10), 3198-3206 (2020). 
30. E. A. Genina, A. N. Bashkatov, and V. V. Tuchin, "Optical clearing of human dura mater by glucose solutions," Journal of Biomedical Photonics \& Engineering 3(1), 010309 (2017).

31. E. C. Cheshire, R. D. G. Malcomson, S. Joseph, M. G. B. Biggs, D. Adlam, and G. N. Rutty, "Optical clearing of the dura mater using glycerol: a reversible process to aid the post-mortem investigation of infant head injury," Forensic Science, Medicine, and Pathology 11(3), 395-404 (2015).

32. S. Mahmoodkalayeh, M. A. Ansari, and V. V. Tuchin, "Head model based on the shape of the subject's head for optical brain imaging," Biomedical Optics Express 10(6), 2795-2808 (2019).

33. A. N. Bashkatov, E. A. Genina, V. I. Kochubey, and V. V. Tuchin, "Optical properties of human cranial bone in the spectral range from 800 to $2000 \mathrm{~nm}$," Proceeding of SPIE 6163, 616310 (2006).

34. E. A. Genina, A. N. Bashkatov, and V. V. Tuchin, "Optical Clearing of Cranial Bone," Advances in Optical Technologies, 267867 (2008).

35. N. Ugryumova, S. J. Matcher, and D. P. Attenburrow, "Measurement of bone mineral density via light scattering," Physics in Medicine and Biology 49(3), 469-483 (2004).

36. M. Firbank, M. Hiraoka, M. Essenpreis, and D. T. Delpy, "Measurement of the optical properties of the skull in the wavelength range 650-950 nm," Physics in Medicine and Biology 38(4), 503-510 (1993).

37. Y. Zhang, C. Zhang, X. Zhong, and D. Zhu, "Quantitative evaluation of SOCS-induced optical clearing efficiency of skull," Quantitative Imaging in Medicine and Surgery 5(1), 136-142 (2015).

38. A. Yu. Sdobnov, J. Lademann, M. E. Darvin, and V. V. Tuchin, "Methods for optical skin clearing in molecular optical imaging in dermatology," Biochemistry (Moscow) 84(1), 144-158 (2019).

39. Y. J. Zhao, T. T. Yu, C. Zhang, Z. Li, Q. M. Luo, T. H. Xu, and D. Zhu, "Skull optical clearing window for in vivo imaging of the mouse cortex at synaptic resolution," Light: Science \& Applications 7(9), 17153 (2018).

40. H. Soleimanzad, H. Gurden, and F. Paina, "Optical properties of mice skull bone in the 455- to 705-nm range," Journal of Biomedical Optics 22(1), 010503 (2017).

41. I. Nishidate, C. Mizushima, K. Yoshida, S. Kawauchi, S. Sato, and M. Sato, "In vivo estimation of light scattering and absorption properties of rat brain using a single-reflectance fiber probe during cortical spreading depression," Journal of Biomedical Optics 20(2), 27003 (2015).

42. I. Nishidate, C. Mizushima, K. Yoshida, S. Kawauchi, S. Sato, and M. Sato, "In vivo estimation of light scattering and absorption properties of rat brain using single reflectance fiber probe during cortical spreading depression," Proceeding of SPIE 8578, 85782Y (2013).

43. J. D. Johansson, "Spectroscopic method for determination of the absorption coefficient in brain tissue," Journal of Biomedical Optics 15(5), 0570059 (2010).

44. X. Fang, B. Pan, W. Liu, Z. Wang, and T. Li, "Effect of scalp hair follicles on NIRS quantification by Monte Carlo simulation and Visible Chinese Human dataset," IEEE Photonics Journal 10(5), 3901110 (2018).

45. J. Tremblay, E. Martinez-Montes, P. Vannasing, D. Nguyen, M. Sawan, F. Lepore, and A. Gallagher, "Comparison of source localization techniques in diffuse optical tomography for fNIRS application using a realistic head model," Biomedical Optics Express 9(7), 2994-3016 (2018).

46. L. Wu, Y. Lin, and T. Li, "Effect of human brain edema on light propagation: a Monte Carlo modeling based on the Visible Chinese Human dataset," IEEE Photonics Journal 9(5), 6101810 (2017).

47. J. Herrera-Vega, S. Montero-Hernandez, I. Tachtsidis, C. G. Trevino-Palacios, and F. Orihuela-Espina, "Modelling and validation of diffuse reflectance of the adult human head for fNIRS: scalp sub-layers definition," Proceeding of SPIE 10572, 1057206 (2017).

48. N. Davoodzaden, M. S. Cano-Velazquez, C. R. Jonak, D. L. Halaney, D. K. Binder, J. Hernandez-Cordero, and G. Aguilar, "Theranostic cranial implant for hyperspectral light delivery and microcirculation imaging without scalp removal," bioRxiv 10(26), (2019).

49. M. M. Koletar, A. Dorr, M. E. Brown, J. McLaurin, and B. Stefanovic, "Refinement of a chronic cranial window implant in the rat for longitudinal in vivo two-photon fluorescence microscopy of neurovascular function," Scientific Reports 9(12), 5499 (2019).

50. N. Kunori, I. Takashima, “An implantable cranial window using a collagen membrane for chronic voltage-sensitive dye imaging," Micromachines 10(11), 789 (2019).

51. M. S. Cano-Velazquez, N. Davoodzadeh, D. Halaney, C. R. Jonak, D. K. Binder, J. Hernandez-Cordero, and G. Aguilar, "Enhanced near infrared optical access to the brain with a transparent cranial implant and scalp optical clearing," Biomedical Optics Express 10(7), 3369-3379 (2019).

52. N. Davoodzadeh, M. S. Cano-Velazquez, D. L. Halaney, C. R. Jonak, D. K. Binder, and G. Aguilar, "Evaluation of a transparent cranial implant as a permanent window for cerebral blood flow imaging," Biomedical Optics Express 9(10), 4879-4892 (2018).

53. S. Ma, L. Ukkonen, L. Sydanheimo, and T. Bjorninen, "Robustness evaluation of split ring resonator antenna system for wireless brain care in semi-anatomical ellipsoid head model," Applied Computational Electromagnetics Society Journal 33(9), 8 (2018).

54. J. D. Johansson, K. Wardell, "Intracerebral quantitative chromophore estimation from reflectance spectra captured during deep brain stimulation implantation," Journal of Biophotonics 6(5), 435-445 (2013). 
55. E. Zinchenko, N. Navolokin, A. Shirokov, B. Khlebtsov, A. Dubrovsky, E. Saranceva, A. Abdurashitov, A. Khorovodov, A. Terskov, A. Mamedova, M. Klimova, I. Agranovich, D. Martinov, V. Tuchin, O. SemyachkinaGlushkovskaya, and J. Kurts, "Pilot study of transcranial photobiomodulation of lymphatic clearance of beta-amyloid from the mouse brain: breakthrough strategies for nonpharmacologic therapy of Alzheimer's disease," Biomedical Optics Express 10(8), 4003-4017 (2019).

56. K. Nowak, E. Mix, J. Gimsa, U. Strauss, K. K. Sriperumbudur, R. Benecke, and U. Gimsa, "Optimizing a rodent model of Parkinson's disease for exploring the effects and mechanisms of deep brain stimulation," Parkinson's Disease, 414682 (2011).

57. S. Mamani, L. Shi, D. Nolan, and R. Alfano, "Majorana vortex photons a form of entangled photons propagation through brain tissue," Journal of Biophotonics 12(10), e201900036 (2019).

58. S. Mamani, L. Shi, T. Ahmed, R. Karnik, A. Rodriguez-Contreras, D. Nolan, and R. Alfano, "Transmission of classically entangled beams through mouse brain tissue," Journal of Biophotonics 11(12), e201800096 (2018).

59. A. Gerega, D. Milej, W. Weigl, M. Kacprzak, and A. Liebert, "Multiwavelength time-resolved near-infrared spectroscopy of the adult head: assessment of intracerebral and extracerebral absorption changes," Biomedical Optics Express 9(7), 2974-2993 (2018).

60. J. T. Alander, O. M. Villet, T. Pätilä, I. S. Kaartinen, M. Lehecka, T. Nakaguchi, T. Suzuki, and V. Tuchin, "Review of Indocyanine Green Imaging in Surgery," Chapter 4 in Fluorescence Imaging for Surgeons: Concepts and Applications, F. D. Dip, T. Ishizawa, N. Kokudo, R. Rosenthal (eds.), Springer International Publishing Switzerland, 35-53 (2015).

61. F. Salehpour, P. Cassano, N. Rouhi, M. R. Hamblin, L. D. Taboada, F. Farajdokht, and J. Mahmoudi, "Penetration profiles of visible and near-infrared lasers and light-emitting diode light through the head tissues in animal and human species: a review of literature," Photobiomodulation, Photomedicine, and Laser Surgery 37(10), 581-595 (2019). 